OPEN ACCESS

Edited by:

Miklos Fuzi,

Semmelweis University, Hungary

Reviewed by:

Ruth M. Hall,

The University of Sydney, Australia

Hong-Yu Ou,

Shanghai Jiao Tong University, China

${ }^{*}$ Correspondence:

János Kiss

kiss.janos@abc.naik.hu

Benoit Doublet

benoit.doublet@inra.fr

Specialty section: This article was submitted to Antimicrobials, Resistance and Chemotherapy,

a section of the journal

Frontiers in Microbiology

Received: 13 November 2018 Accepted: 20 February 2019

Published: 06 March 2019

Citation:

Kiss J, Szabó M, Hegyi A, Douard G, Praud K, Nagy I, Olasz F, Cloeckaert A and Doublet B (2019) Identification and Characterization of oriT and Two Mobilization Genes Required for Conjugative Transfer of Salmonella Genomic Island 1

Front. Microbiol. 10:457 doi: 10.3389/fmicb.2019.00457

\section{Identification and Characterization of oriT and Two Mobilization Genes Required for Conjugative Transfer of Salmonella Genomic Island 1}

\author{
János Kiss ${ }^{*}$, Mónika Szabó1, Anna Hegyi1,2, Gregory Douard ${ }^{2}$, Karine Praud ${ }^{2}$, \\ István Nagy', Ferenc Olasz' ${ }^{1}$, Axel Cloeckaert ${ }^{2}$ and Benoît Doublet ${ }^{2 *}$ \\ ${ }^{1}$ National Agricultural Research and Innovation Centre, Agricultural Biotechnology Institute, Gödölllo, Hungary, ${ }^{2}$ ISP, Institut \\ National de la Recherche Agronomique, Université de Tours, UMR 1282, Nouzilly, France
}

The integrative mobilizable elements of SGl1-family considerably contribute to the spread of resistance to critically important antibiotics among enteric bacteria. Even though many aspects of SGl1 mobilization by IncA and IncC plasmids have been explored, the basic transfer elements such as oriT and self-encoded mobilization proteins remain undiscovered. Here we describe the mobilization region of SGl1 that is well conserved throughout the family and carries the oriT SGl1 $_{1}$ and two genes, $m p s A$ and mpsB (originally annotated as S020 and S019, respectively) that are essential for

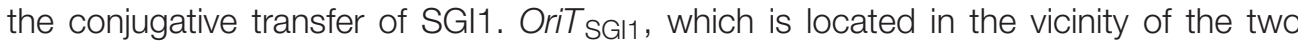
mobilization genes proved to be a 125-bp GC-rich sequence with several important inverted repeat motifs. The mobilization proteins MpsA and MpsB are expressed from a bicistronic mRNA, although MpsB can be produced from its own mRNA as well. The protein structure predictions imply that MpsA belongs to the lambda tyrosine recombinase family, while MpsB resembles the $\mathrm{N}$-terminal core DNA binding domains of these enzymes. The results suggest that MpsA may act as an atypical relaxase, which needs MpsB for SGl1 transfer. Although the helper plasmid-encoded relaxase proved not to be essential for SGl1 transfer, it appeared to be important to achieve the high transfer rate of the island observed with the IncA/IncC-SGl1 system.

Keywords: salmonella genomic island 1, integrative mobilizable element, IncA/C plasmids, origin of transfer (oriT), horizontal gene transfer, mobile genetic element (MGE), antibiotic resistance (AR)

\section{INTRODUCTION}

Conjugation is a widespread mechanism of horizontal gene transfer among naturally occurring plasmids and genomic islands. During conjugation, DNA is transferred from donor to recipient through a close cell-to-cell contact. Based on Gram-negative plasmid models, the process starts with the assembly of a multi-protein complex called the relaxosome around the origin of transfer (oriT). OriT is a cis-acting DNA region that is required for initiation of the transfer. The key enzyme of transfer initiation is the relaxase, which cuts either strand of oriT DNA at the nic site. The relaxase remains covalently bound to the $5^{\prime}$-end of the cleaved strand, which is subsequently delivered to 
the recipient across the membrane-associated DNA transport machinery, the type IV secretion system (T4SS). Initiation of conjugation often requires several auxiliary proteins implicated in the relaxosome (De La Cruz et al., 2010; Bellanger et al., 2014). The relaxosome is then recruited to the T4SS with the assistance of the membrane-associated coupling proteins (T4CP), which binds the cognate T4SS and the relaxosome complex through the relaxase or auxiliary proteins (Llosa and Alkorta, 2017). Based on phylogenetic analyses of relaxases, conjugative systems have been classified into eight major MOB families, however, numerous unclassified systems have also been reported (Garcillán-Barcia et al., 2009; Bellanger et al., 2014; Li et al., 2018). Some of these families include plasmids and mobile genomic islands (MGIs) as well (e.g., $\mathrm{MOB}_{\mathrm{H}}, \mathrm{MOB}_{\mathrm{C}}, \mathrm{MOB}_{\mathrm{P}}, \mathrm{MOB}_{\mathrm{V}}, \mathrm{MOB}_{\mathrm{T}}$ ), indicating the relationship of the conjugative systems of the two groups of mobile elements.

In addition to resistance plasmids, MGIs are the major players in dissemination of multidrug resistance (MDR) phenotype among bacteria. MGIs provide selective advantages to their host by carrying resistance-, pathogenicity-, metabolic pathwayor symbiosis-related genes. After acquisition, MGIs integrate into the host chromosome by site-specific-, transposition- or homologous recombination to ensure their maintenance and vertical transmission (Bellanger et al., 2014). MGIs are classified into two major groups: integrative conjugative elements (ICEs, some of them were formerly known as conjugative transposons, (Liu et al., 2019) and integrative mobilizable elements (IMEs). In addition to the chromosomal excision/integration ability, ICEs encode for their own conjugation system and are fully autonomous in horizontal transfer (Carraro and Burrus, 2014), while IMEs have a limited set of transfer genes and thus require the presence of other conjugative helper elements to hijack their transfer systems (Douard et al., 2010, Daccord et al., 2013; Bellanger et al., 2014).

Many studies about MGI mobilization unravel their integration and excision reactions. However, the mechanism of their conjugation is less investigated, and their transfer genes are mostly identified by their similarities to those of conjugative plasmids. Like all conjugative and mobilizable plasmids, MGIs also carry their own oriT sequence (Bellanger et al., 2014). Most oriT sequences have been identified in conjugative plasmids, where they are often located adjacent to genes of the relaxosome components (e.g., in RP4, R388, R6K, pCW3, pIP501). Although conserved sequence motifs have been found in oriTs close to the nic site of IncP $\alpha, \mathrm{Ti}, \mathrm{Ri}, \mathrm{R} 64$ and pTF-FC2 plasmids (Pansegrau and Lanka, 1991), oriTs are generally diverse sequences [oriTDB (Li et al., 2018)], which frequently contain inverted repeat (IR) motifs and AT-rich regions [F (Frost et al., 1994), R6K (Avila et al., 1996), IncP $\alpha$ (Fürste et al., 1989),(Pansegrau et al., 1994), pAD1 (Francia and Clewell, 2002), pAM373 (Francia and Clewell, 2002), pCW3 (Wisniewski et al., 2016) and IncA and IncC plasmids (Hegyi et al., 2017)]. Transfer systems of several ICEs have also been analyzed in details and the oriT has been identified in Tn916 (Jaworski and Clewell, 1995), Tn4445 (Smith and Parker, 1998), ICEBs1 (Lee and Grossman, 2007), SXT/R391 (Ceccarelli et al., 2008), ICEclc (Miyazaki and Van Der Meer, 2011), ICEhptfs4 (formerly referred as tfs4)
(Grove et al., 2013) and the related IMEs mobilized by SXT (Daccord et al., 2010, 2013; Li et al., 2018). Although all oriTs contain several IR motifs, no extensive sequence similarities can be observed between oriTs of unrelated elements. In contrast, IMEs hijacking the same helper elements have similar oriTs to each other and to that of the helper (Daccord et al., 2010). Most conjugative plasmids and MGIs have a single oriT, however, in some cases two separate and functional oriTs have been identified [pAD1 (Francia et al., 2001; Francia and Clewell, 2002), R6K (Avila et al., 1996), ICEclc (Miyazaki and Van Der Meer, 2011)].

Among the few well-studied families of MGIs, the Salmonella genomic island 1 (SGI1) is one of the largest and most diverse IME family, which considerably contribute to spreading MDR and especially the resistance to critically important antibiotics such as extended-spectrum $\beta$-lactams or carbapenems. The prototype of SGI1 has been described in a multidrug resistant clone of Salmonella enterica serovar Typhimurium DT104 (Boyd et al., 2000), which emerged during the mid-1980s (Threlfall et al., 1994) and spread worldwide. The common multidrug resistance phenotype (ACSSuT) of this epidemic clone is conferred by the 42.4 -kb SGI1, which contains 44 predicted open reading frames (ORFs) and carries a ca. $15 \mathrm{~kb}$ complex In4-type integron structure called In104 (Figure 1A). In104 is inserted near the $3^{\prime}$-end of the SGI1 backbone (Boyd et al., 2001) and flanked by 25-bp imperfect inverted repeats. In the prototype SGI1 this gene cluster contains two class 1 integron structures with gene cassettes aadA2 and bla $a_{\mathrm{CARB}-2}\left(\right.$ bla $\left.a_{\mathrm{PSE}-1}\right)$, respectively, other resistance genes (tet $A(\mathrm{G})$ and floR) occurring independently of the integrons, IS elements (ISCR3, IS6100) and some additional genes of unknown function. Loss, gain or exchange of antibiotic resistance genes have occurred mainly in In104 by exchanges of resistance gene cassettes, homologous recombinations or IS-induced rearrangements, which led to the emergence of known SGI1 variants (SGI1-A to SGI1Z12, SGI2) identified in numerous S. enterica serovars, Proteus mirabilis strains and Morganella morganii (Levings et al., 2005, 2008; Doublet et al., 2008; Siebor and Neuwirth, 2011, 2014; Schultz et al., 2017). Recently, SGI1-related elements have been described in several other species such as PGI1 and PGI2 in P. mirabilis and AGI1 in Acinetobacter baumannii (Siebor and Neuwirth, 2014; Hamidian et al., 2015). Other uncharacterized SGI1-related elements are found in Aeromonas veronii, Vibrio cholerae (Johnson et al., 2015), Vibrio mimicus, Shewanella and Enterobacter spp. (Supplementary Figure S1).

The SGI1-family islands are typical IMEs, as they integrate autonomously into or excise from the bacterial chromosome specifically at the $3^{\prime}$-end of trmE gene (formerly thdF and also called $m n m E$ ), but they do not encode all the genes necessary for their self-transfer, and require the conjugation system of plasmids of the incompatibility groups $\mathrm{A}$ or $\mathrm{C}$ for their horizontal transfer (Doublet et al., 2005, Daccord et al., 2010). The IncC family with hundreds of fully sequenced plasmids includes broad host range, single-copy conjugative plasmids implicated in MDR dissemination, while IncA group contains only one sequenced member, RA1, which has a backbone closely related to that of IncC plasmids, but proved to be compatible with them (Datta and Hedges, 1973; Ambrose et al., 2018). Their 
A

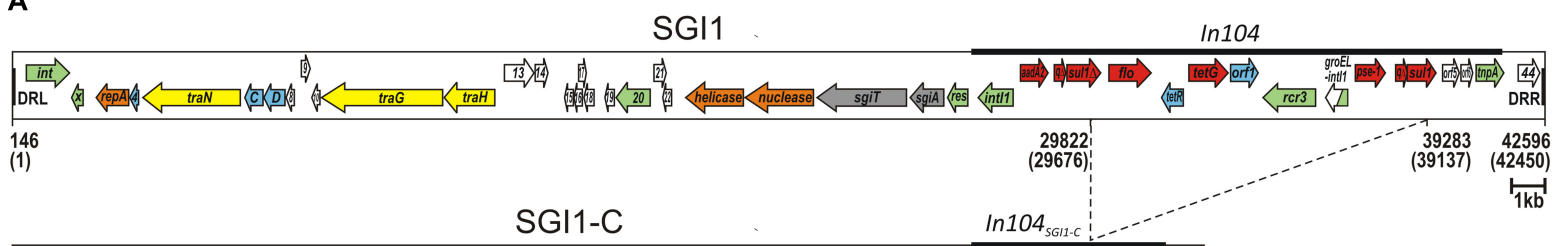
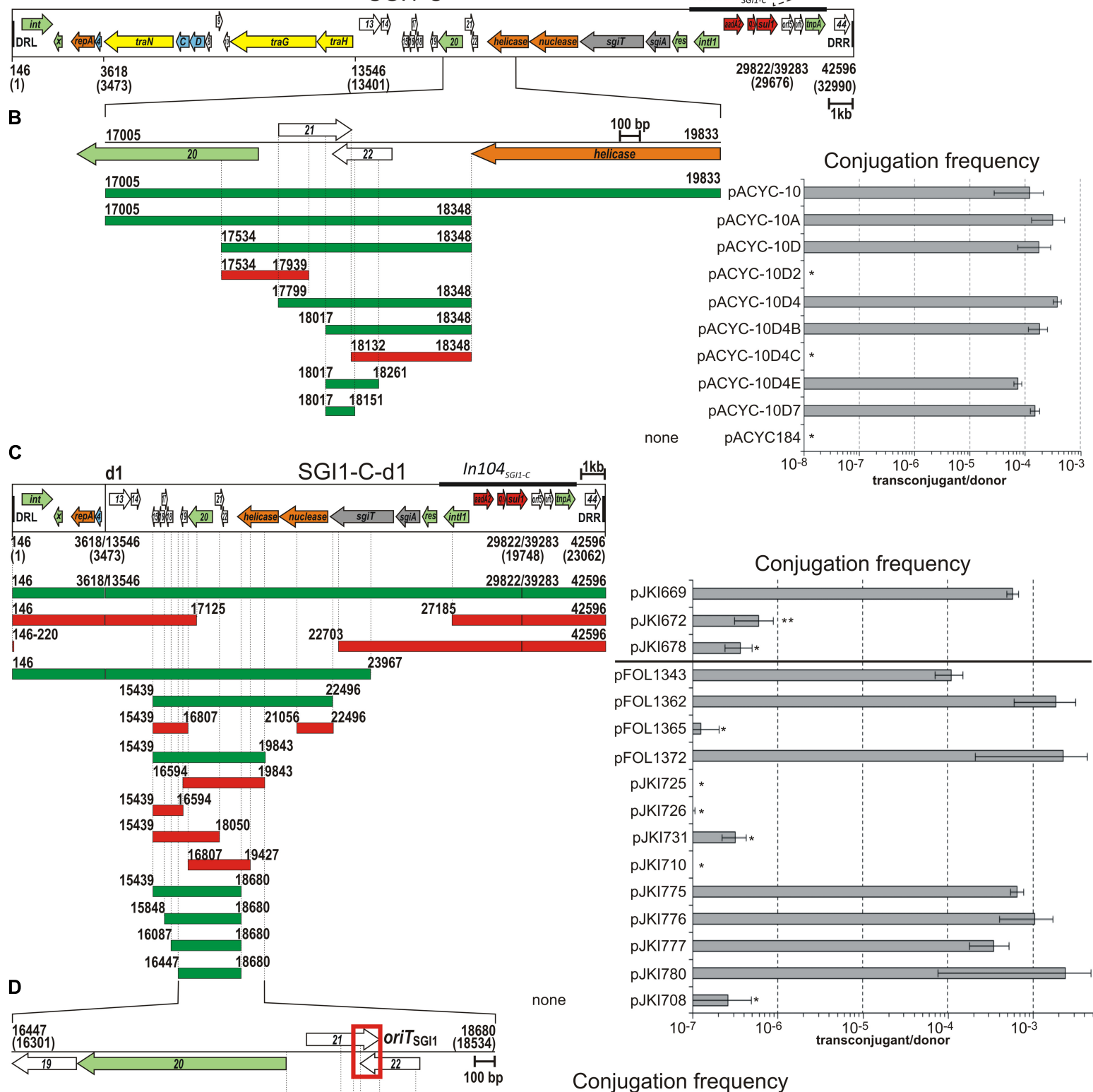

none

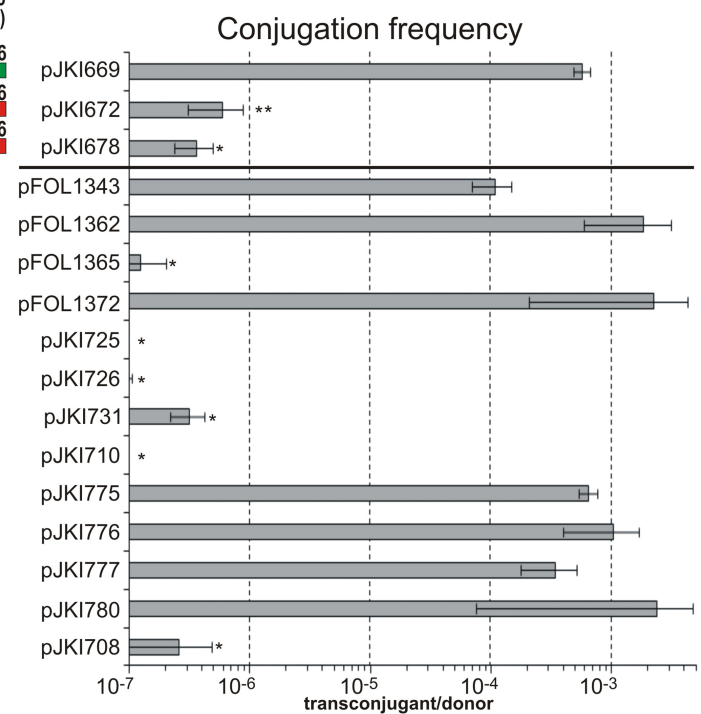

16447

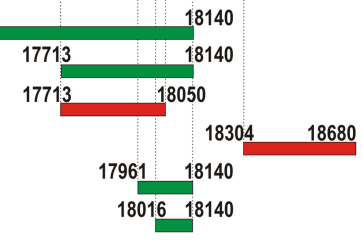

Conjugation frequency

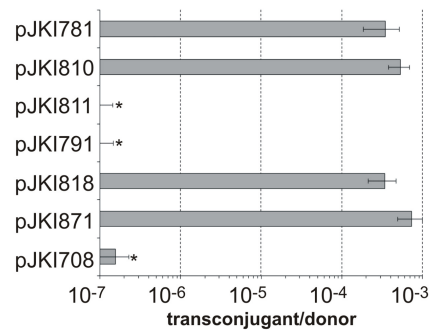

FIGURE 1 | Continued 


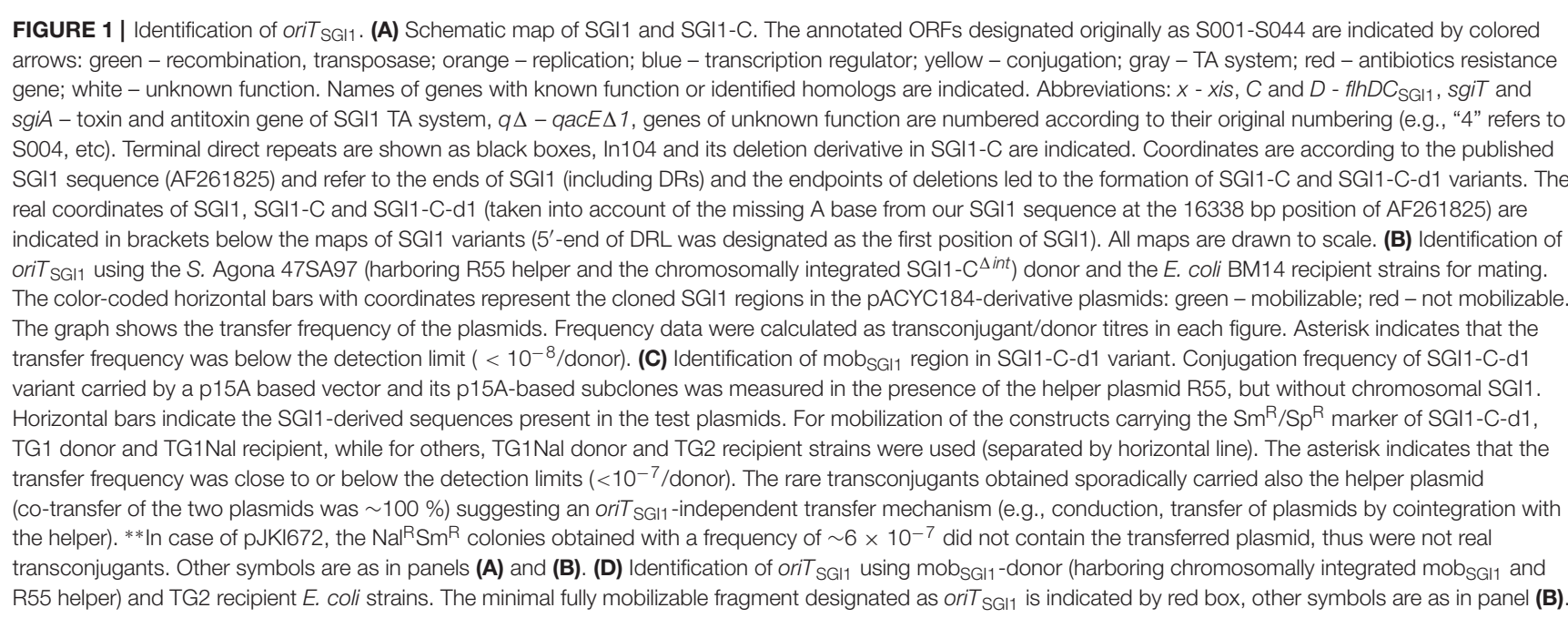

conjugative system, which is related to that of SXT/R391 ICEs (Wozniak et al., 2009; Poulin-Laprade et al., 2015), has been classified into the $\mathrm{MOB}_{\mathrm{H} 12}$ group (Garcillán-Barcia et al., 2009). SGI1 is mobilized by IncA and IncC (hereafter are referred as IncA/C) plasmids such as RA1, R55, R16a, IP40a, pVCR94 or pRMH760 (Douard et al., 2010; Kiss et al., 2012; Carraro et al., 2014; Harmer et al., 2016), however, other unrelated IMEs (e.g., MGIVmil of V. mimicus) have also been reported to utilize these plasmids as mobilization helpers (Carraro et al., 2014, 2016). Expression of the conjugation apparatus of IncA/C plasmids are controlled by the FlhDC-family master activator, AcaCD (Carraro et al., 2014), which is required for the transfer of both the plasmid and the mobilized IMEs (Carraro et al., 2014; Kiss et al., 2015). The plasmid-encoded AcaCD triggers the excision of SGI1 through the activation of expression of the SGI1-encoded recombination directionality factor Xis (Carraro et al., 2014; Kiss et al., 2015), but has no effect on the expression of the site-specific recombinase Int (Kiss et al., 2015) required for both the excision and integration (Doublet et al., 2005). After SGI1 transfer, the island efficiently integrates into the attB site in trmE.

Recent studies have indicated a more complex crosstalk between SGI1 and IncA/C plasmids. In addition to the xis promoter, four other AcaCD-responsive promoters have been identified on SGI1 (Carraro et al., 2014; Murányi et al., 2016). Moreover, the island also encodes its own functional FlhDC-

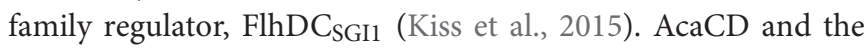

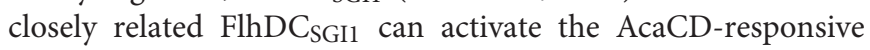
promoters on both SGI1 and the conjugation regulon of IncA/C plasmids (Murányi et al., 2016). The SGI1-encoded transfer proteins TraN, TraG, and TraH, whose expression is also under AcaCD-control, interact with the plasmid encoded homologs and promote SGI1 transfer at the expense of the helper plasmid (Carraro et al., 2017). Furthermore, SGI1 and IncA/C plasmids encode for incompatibility functions impeding stable co-habitation of the partners in the same host (Harmer et al., 2016; Huguet et al., 2016). Destabilization of SGI1 by IncA/C plasmids seems to be based on triggering of SGI1 excision by
AcaCD (Kiss et al., 2015), however, the similar effect of SGI1 on the helper plasmids appear more complex and poorly understood (Harmer et al., 2016).

Even though many aspects of the SGI1-IncA/C dual system have been studied, the basic transfer functions of SGI1, i.e., the oriT and the putative self-encoded mobilization proteins remain undiscovered. The three SGI1-encoded tra genes, traN, traG and traH, have an important but not essential role in the transfer of the island (Carraro et al., 2017; Kiss et al., 2012). Other ORFs of SGI1 do not show sequence similarity to known tra genes and relaxases, including that of IncA/C plasmids, thus, the implication of an atypical relaxase in SGI1 transfer can not be excluded. Similarly, oriT of SGI1 can not be identified on the basis of sequence homology with oriT of IncA/C plasmids (Hegyi et al., 2017), despite the fact that the plasmid-encoded relaxase is required for efficient SGI1 transfer. In the present work, we identify the mobilization region of SGI1 carrying the cisacting oriT sequence and two predicted ORFs encoding proteins that are essential for the conjugative transfer of the island. The minimal oriT sequence, ori $T_{\mathrm{SGI} 1}$, has been characterized, and the importance of its inverted repeat (IR) motifs for the transfer was examined. Comparative analysis of SGI1-related elements confirmed that the mobSI1 region is well conserved throughout the family, strengthening its importance in the horizontal transfer of SGI1. The predicted structure and possible function of the two transfer proteins identified have also been discussed.

\section{MATERIALS AND METHODS}

\section{Microbial Techniques and DNA Procedures}

Relevant features of the bacterial strains and plasmids are listed in Supplementary Tables S1, S2, respectively. Bacterial strains were maintained at $-80^{\circ} \mathrm{C}$ in $\mathrm{LB}$ broth containing $30 \%$ glycerol and were routinely grown in Luria-Bertani (LB) broth at $37^{\circ} \mathrm{C}$ supplemented with the appropriate antibiotics used at a final concentration as follows: ampicillin (Ap) 
$150 \mu \mathrm{g} / \mathrm{ml}$, chloramphenicol (Cm) $20 \mu \mathrm{g} / \mathrm{ml}$, kanamycin (Km) $30 \mu \mathrm{g} / \mathrm{ml}$, spectinomycin (Sp) $50 \mu \mathrm{g} / \mathrm{ml}$, streptomycin (Sm) $50 \mu \mathrm{g} / \mathrm{ml}$, nalidixic acid (Nal) $20 \mu \mathrm{g} / \mathrm{ml}$, gentamicin $(\mathrm{Gm})$ $25 \mu \mathrm{g} / \mathrm{ml}$, tetracycline (Tc) $10 \mu \mathrm{g} / \mathrm{ml}$, sodium azide (Az) $500 \mu \mathrm{g} / \mathrm{ml}$. Standard molecular biology procedures were carried out according to Sambrook et al. (1989). Detailed methodology of plasmid constructions is described in Supplementary Methods. Test/colony PCRs were performed using Dream Taq polymerase (Thermo Fisher Scientific) as previously described (Kiss et al., 2012). The amplicons for cloning were amplified with Phusion (Thermo Fisher Scientific) or Pwo (Roche) polymerases and sequenced on ABI Prism 3100 Genetic Analyzer (PerkinElmer). Oligonucleotide primers used in this work are listed in Supplementary Table S3. Primers annealing to SGI1 or R55 were designed according to the published sequence of SGI1 (GenBank: AF261825) and R55 (GenBank: JQ010984).

The prototype SGI1 carries five functional resistance genes, which makes difficult to set up mating or $\mathrm{KO}$ mutagenesis experiments, thus for mating assays, cloning or mutagenesis we used the $\mathrm{Sm}^{\mathrm{R}} / \mathrm{Sp}^{\mathrm{R}}, \mathrm{Sul}^{\mathrm{R}}$ derivative, SGI1-C (Figure 1A), which was previously shown to have the same mobilization properties as SGI1 (Kiss et al., 2012).

The $\beta$-galactosidase assay was performed in five replicates according to Miller (1972) except that the cultures were grown at $37^{\circ} \mathrm{C}$ to an $\mathrm{OD}_{600} \sim 0.3$ in $\mathrm{LB}$ both and diluted at a ratio of 1:1 with $\mathrm{Z}$ buffer.

Primer extension reactions were carried out as described (Murányi et al., 2016) using the $\beta$-gal tester plasmids pMSZ1017 and pMSZ948 carrying the upstream regions of S019 and S020, respectively.

\section{Conjugation Assays}

Deletion derivatives of SGI1-C-d1 in pJKI669 were generated by enzymatic digestion and re-ligation and introduced into Escherichia coli TG1/R55. Other smaller SGI1 backbone fragments were amplified by PCR, cloned into pJKI708 vector and transformed into TG1Nal/R55 strain. The resulting TG1 or TG1Nal donor strains harboring one of the test plasmids along with R55 helper were then used in standard mating assays with TG1Nal or TG2 recipients, respectively, as described previously (Kiss et al., 2012).

Mating with $S$. Agona donor strains were performed by mixing mid-log phase $\left(\mathrm{OD}_{600}=0.5\right)$ cultures of the donor strains harboring SGI1 and R55 w/o pACYC184-derivative test plasmids and the sodium azide-resistant E. coli recipient strain BM14 in a ratio of $1: 1$. The mix was incubated overnight at $37^{\circ} \mathrm{C}$ without shaking and then cells were streaked on appropriate selective SS agar plates. Transconjugants were selected on sodium azide combined with antibiotics depending on the transferred element: SGI1 - Sm, R55 - Km and the pACYC184 derivatives - Tc. The transfer frequencies were calculated as the ratio of transconjugant and donor titers. Complementation assays for identification of promoter regions of S020 and S019 were carried out as described (Kiss et al., 2012), except that mating was done with TG90 recipient in $2 \mathrm{YT}$ plates for $6 \mathrm{~h}$ to reduce the growth of the donor over the recipient cells.
IncC plasmid R16a was used as helper plasmid in some mating assays due to its more suitable resistance markers. It has previously been shown to be as effective mobilizer of SGI1 as R55 (Douard et al., 2010). In mobilization tests of the S019 and S020 KO (Knock-Out) mutant or WT mobsGI1-containing plasmids (pJKI772, pJKI737, pFOL1372) or SGI1-C by R16aWT or R16a ${ }^{\Delta \text { TraI }}$ helper plasmids, the E. coli donor strains TG1Nal or TG1Nal::SGI1-C were used with TG90 recipient. Mobilization of SGI1-C ${ }^{\Delta o r i T}$ and pMNI41 by $\mathrm{R}^{\Delta 5^{\Delta \mathrm{Tn}} 6187}$ helper plasmid was assayed by mating of E. coli TG1Nal donor and E. coli TG90 recipient starins. In case of low frequency transfer, rare transconjugants were detected by spreading $100 \mu \mathrm{l}$ (instead of dropping $5 \mu \mathrm{l}$ ) of undiluted bacterial suspension obtained from the mating LB plates onto the appropriate selective plates.

\section{Targeted Gene KO Experiments}

The PCR fragments for KO mutagenesis of S019, S020, S022 and ori $_{\mathrm{SGI}}$ were amplified from pKD3 template plasmid using primers delS019for-delS019rev, delS020for-delS020rev, delS022for-delS022rev, and deloriTfor-deloriTrev, respectively, (Supplementary Table S3). For promoting the gene replacement recombination between the targeted region and the respective KO PCR fragment, $\lambda$ Red recombinase was expressed from the plasmid pKD46 or its $\mathrm{Tc}^{\mathrm{R}}$ derivative plasmid pJKI842 using 1\% $\mathrm{L}$-arabinose as inductor at $30^{\circ} \mathrm{C}$ for $1.5 \mathrm{~h}$. The $\mathrm{Cm}^{\mathrm{R}}$ cassette was removed from the chromosomal $\mathrm{KO}$ alleles by expressing the FLP recombinase from the thermo-inducible expression plasmid pCP20 (Datsenko and Wanner, 2000), or by digestion with $\mathrm{XbaI}$ (present in FRT sites) followed by religation in the case of pFOL1372 derivatives. The plasmids having temperaturesensitive pSC101 replication system were maintained and cured at 30 and $42^{\circ} \mathrm{C}$, respectively. In the resulting $\mathrm{KO}$ mutants, a short region was replaced with 83 -bp sequence deriving from the PCR template plasmid pKD3. The replacements near the $5^{\prime}$-end of S019, S020, and S022 generated early stop codons in the ORFs.

The KO PCR fragment for the scarless deletion (Kolisnychenko et al., 2002) of Tn6187 in R55 was amplified from pJKI1023 template plasmid using primers R55-dTn6187ABforR55-dTn6187Crev. After the $\lambda$ Red-induced recombination/gene replacement, the $\mathrm{Sm}^{\mathrm{R}}$ marker gene was eliminated from the resulting $\mathrm{R} 55^{\Delta \mathrm{Tn} 6187}:: \mathrm{Sm}^{\mathrm{R}}$ plasmid by DSB-stimulated recombinational repair process induced by I-SceI cleavage. Expression of I-SceI from pMSZ934 was induced by $30 \mu \mathrm{g} / \mathrm{ml}$ chlortetracycline (cTc) $\mathrm{O} / \mathrm{N}$ at $30^{\circ} \mathrm{C}$ in $\mathrm{LB}+\mathrm{Cm}+\mathrm{Ap}$. The $\mathrm{Sm}^{\mathrm{S}}$ clones were selected by replica plating and the scarless site was amplified and sequenced. To demonstrate that deletion of Tn6187 did not affect the conjugation properties of R55, mating assay was conducted with TG1Nal donor and TG90Nal $\left(\mathrm{Tc}^{\mathrm{R}}\right)$ recipient strains, where $\mathrm{R} 55$ and its $\Delta \mathrm{Tn} 6187$ derivative showed similar transfer frequencies $\left(2.9 \pm 1.0 \times 10^{-1}\right.$ and $2.5 \pm 0.8 \times 10^{-1}$, respectively).

\section{Construction of mobsGI1-Donor Strain}

For chromosomal integration of the 16447-18680 bp SGI1 regions, the pLOFKm (Herrero et al., 1990) derivative pJKI796 was introduced into $E$. coli S17-1 $\lambda$ pir strain, which allows the replication and transfer of R6K-based plasmids carrying 
the oriT of RK2. One of the S17-1 $\lambda$ pir/pJKI796 transformant colonies was used as donor in a standard mating (Kiss et al., 2012) with TG1Nal recipient. The miniTn10::mobsGI$\mathrm{Km}^{\mathrm{R}}$ chromosomal integrants were selected on $\mathrm{LB}+\mathrm{Nal}+\mathrm{Km}$ plates at $37^{\circ} \mathrm{C} \mathrm{O} / \mathrm{N} . \mathrm{Km}^{\mathrm{R}} \mathrm{Nal}^{\mathrm{R}}$ transconjugants were streaked twice onto $\mathrm{LB}+\mathrm{Nal}+\mathrm{Km}$ plates and tested for $\mathrm{Ap}^{\mathrm{S}}$ phenotype indicating the loss of plasmid backbone of pJKI796 (conservative transposition of the miniTn 10 unit). Then, R55 was transferred into the resulting strain from TG1/R55 in a standard mating. Transconjugants were selected on $\mathrm{LB}+\mathrm{Nal}+\mathrm{Cm}$ plates at $37{ }^{\circ} \mathrm{C}$ $\mathrm{O} / \mathrm{N}$, and streaked twice on $\mathrm{LB}+\mathrm{Nal}+\mathrm{Cm}$ plates to get rid of donor contamination.

\section{Bioinformatics}

Sequence alignments were generated using the MultAlin interface $^{1}$ (Corpet, 1988). Promoter motifs were predicted by BPROM $^{2}$ (Solovyev and Salamov, 2011). For searching protein motifs MOTIF Search ${ }^{3}$ was used. Protein structure modeling was conducted using Phyre2 ${ }^{4}$ (Kelley et al., 2015), Swiss-Model ${ }^{5}$ (Arnold et al., 2006) and PSIPRED ${ }^{6}$ (Buchan et al., 2013). All homology searches were performed with the NCBI BLAST and DELTA-BLAST server ${ }^{7}$. SGI1-related elements were identified via a nucleotide BLAST search in GenBank using SGI1 backbone as query sequence, which was generated from the reference SGI1 sequence AF261825 by deletion of the flanking non-SGI1 sequences of DRs and In104 region along with one copy of the 5-bp direct repeat (DR associated with integron IRi) delimiting the In 104 gene cluster. Inverted repeat motifs were detected using $\mathrm{mFold}^{8}$ server (Zuker, 2003).

\section{RESULTS}

\section{Identification and Functional Analysis of the SGI1 Transfer Origin Localization of the oriT Sequence in SGI1}

To determine the location of oriT $T_{\text {SGI1 }}, 14$ different segments covering almost the entire SGI1-C backbone (excluding In104) were cloned into the non-mobilizable low-copy number vector pACYC184. These plasmids (pACYC184-1 to pACYC184-14) were introduced into the $S$. enterica serovar Agona strain 47SA97SGI1 ${ }^{\Delta \text { int }}$ harboring the IncC helper plasmid R55 and their mobilization were assessed in mating assays. Nonmobilizable chromosomal SGI1 ${ }^{\Delta i n t}$ was used to provide all (yet unknown) necessary trans mobilization factors and to prevent the mobilization of pACYC184 derivatives along with SGI1 via homologous recombination-mediated cointegration. Unlike other plasmids, pACYC-10 carrying SGI1 sequence from position

\footnotetext{
${ }^{1}$ http://multalin.toulouse.inra.fr/multalin/

${ }^{2}$ https://omictools.com/bprom-tool

${ }^{3}$ http://www.genome.jp/tools/motif/

${ }^{4}$ http://www.sbg.bio.ic.ac.uk/phyre2/html/page.cgi?id=index

${ }^{5}$ https://swissmodel.expasy.org/

${ }^{6}$ http://bioinf.cs.ucl.ac.uk//psipred/

${ }^{7}$ https://blast.ncbi.nlm.nih.gov/Blast.cgi

${ }^{8}$ http://unafold.rna.albany.edu/?q=mfold/DNA-Folding-Form
}

17005 to $19833 \mathrm{bp}$ (Figure 1B) proved to be mobilizable at a comparable frequency $\left(1.2 \pm 1.0 \times 10^{-4}\right)$ to that of the wild-type (WT) SGI1-C (SGI1 positions are given according to the GenBank entry AF261825 used as reference sequence). However, pACYC-10 could not be mobilized in the absence of $\mathrm{SGI}^{\Delta \text { int }}$ in the donor strain (transfer frequency was below the $10^{-8}$ detection limit). This suggested that this SGI1 fragment (the region flanked by ORFs S020 and S023) carried at least the cis-acting oriT sequence of SGI1, while other mobilization functions provided in trans by SGI1 ${ }^{\Delta i n t}$ were also required for mobilization of pACYC-10.

To determine the minimal ori $T_{\mathrm{SGI}}$, the fragment carried by pACYC-10 was progressively reduced in size and assessed for mobilization in similar assays (Figure 1B). The shortest mobilizable clone was pACYC-10D7 carrying a 135-bp SGI1 sequence extending from 18017 to $18151 \mathrm{bp}$, indicating that the fully functional ori $T_{\mathrm{SGI}}$ overlaps the $3^{\prime}$-end of the predicted ORFs S021 and S022. The analysis of 10 transconjugants from each independent positive experiment confirmed the presence of the pACYC derivatives and the absence of the IncC helper plasmid, implying their conjugative mobilization in trans.

\section{Minimal Mobilizable Region of SGI1}

In parallel with the above approach, an alternative method was also applied to determine the entire region carrying all cis- and trans-acting mobilization functions of SGI1. The ca. $23 \mathrm{~kb}$ deletion derivative of SGI1-C (designated SGI1-C-d1, Figure 1C), which was previously shown to be mobilizable by R55 (Kiss et al., 2012) was cloned into a non-mobilizable p15A-based vector (plasmid pJKI669). Deletion derivatives and subclones of pJKI669 were used in mating assays in absence of chromosomal SGI1 in the donor strain. Only the plasmid constructs containing the intact S019-S022 region (position 16447 to $18680 \mathrm{bp}$ ) proved to be mobilizable in trans by R55 at a frequency comparable to the transfer of SGI1-Cd1 from pJKI669 $\left(5.74 \pm 0.93 \times 10^{-4}\right.$, Figure 1C). The shortest mobilizable construct, pJKI780, contained a $2.2 \mathrm{~kb}$ insert spanning SGI1 from the end of S019 to that of S023. Plasmids in which S019 (pJKI710, pJKI725), S020 (pJKI710, pJKI726) or S022 (pJKI731) were partially or entirely missing could not be mobilized. These results suggested that the $2.2 \mathrm{~kb}$ region cloned in pJKI780, hereafter called as mobSGI, carries not only the ori $T_{\mathrm{SGI}}$, but also all genes that are indispensable for SGI1 mobilization.

\section{Identification of the Minimal oriT SGl1 $_{\text {S }}$}

Since further reduction of cloned mobsGI region impaired the transfer of the test plasmid, exact localization of oriT $T_{\mathrm{SGI}}$ and the determination of the minimal functional oriT $T_{\mathrm{SGI}}$ region required a helper strain which provides all mobilization factors in trans similarly, to the $S$. Agona strain $47 \mathrm{SA97SGI1}{ }^{\Delta i n t}$ applied previously for mobilization of pACYC184-derivatives. Thus, a donor strain containing the chromosomally integrated

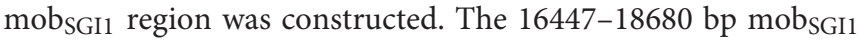
region was integrated into the chromosome of $E$. coli strain TG1Nal using a mini-Tn10 transposon (Herrero et al., 1990), and then the helper plasmid R55 was introduced. The resulting 
"mobsGI1-donor" strain was used to test the mobilization of plasmid subclones carrying progressively shortened fragments of mobsGI1 region by similar method applied above (see Figure 1C). The smallest SGI1 fragment permitting the mobilization of the test plasmid corresponded to positions 17961-18140 of SGI1 (pJKI818, Figure 1D). Based on the alternative methods (Figures 1B,D), we identified a 125-bp sequence corresponding to the 18016-18140 bp fragment of SGI1 (deduced from the overlap of inserts in pJKI818 and pACYC$10 \mathrm{D} 7)$ as the minimal fully active ori $_{\mathrm{SGI}}$ (pJKI871, Figure 1D), which is localized in the $3^{\prime}$-end of ORF S021 including the overlapping part of S022.

\section{Deletion of oriT SGl1 $_{\text {Causes Transfer-Deficiency }}$}

To further examine the ori $T_{\mathrm{SGI} 1}$, we knocked out this segment from SGI1-C using the one-step gene inactivation method. In the conjugation assays, mobilization of SGI1-C $\mathrm{C}^{\Delta \text { oriT }}$ and a $15 \mathrm{~A}-$ based plasmid carrying the 125 -bp ori $T_{\text {SGI1 }}$ sequence (pMNI41) was assessed. For this assay, the R55 ${ }^{\Delta \operatorname{Tn} 6187}$ helper plasmid was used due to its reduced antibiotic resistance spectrum $\left(\mathrm{Flo}^{\mathrm{R}} / \mathrm{Cm}^{\mathrm{R}}, \mathrm{Sul}^{\mathrm{R}}\right)$. While the SGI1-C $\mathrm{C}^{\Delta \text { oriT }}$ mutant proved to be non-mobilizable $\left(<7.3 \pm 2.3 \times 10^{-8}\right)$, the plasmid pMNI41 was mobilized in trans at a frequency of $7.3 \pm 2.9 \times 10^{-3}$, which was comparable to the transfer rate of pJKI871 also containing oriT $T_{\mathrm{SGI}}$ (Figure 1D). This result indicated that deletion of oriT $_{\mathrm{SGI}}$ sequence abolished the SGI1 transfer, while the functions required in trans for mobilization of ori $T_{\mathrm{SGI}^{-}}$carrying plasmid were not affected.

\section{Functional Analysis of Mutations Affecting IR Motifs in oriT SGl1 $_{1}$}

The ori $_{\text {SGI1 }}$ sequence contains an array of inverted repeats (IRs, Figure 2A). For a more detailed analysis the oriT sequence, was further shortened or the repetitive motifs were mutated and the

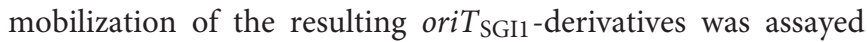
using the mobsGI donor strain as described above. Mutations of the 7-bp perfect GC-rich inverse repeat IR1 resulted in significant reduction of the transfer frequency. Complete or partial deletion of IR1 entirely abolished the plasmid mobilization (pMSZ990, pMSZ989, and pJKI874; Figure 2A). Interestingly, deletion of the 7-bp AT rich tract located upstream of IR1 caused a 15fold reduction of the transfer rate, which was further reduced by factors of 7 and 26 by the single base deletions in IR1R (compare pJKI871, pMSZ997, pMSZ995 and pMSZ991). Similarly, deletion of the right arm of the 19-bp imperfect repeat IR3 caused a 40fold reduction of transfer rate (pJKI873 and pJKI872). In contrast, base changes introduced into the right arm of the 12-bp imperfect repeat IR2, abolishing the putative stem-loop structure, had no detectable effect on the transfer frequency. The two 6-bp GC-rich IRs located upstream of oriT $T_{\text {SGI1 }}(17987-18017$ bp, Figure 2A) seemed to have no or marginal role in oriT function, as their absence did not reduce the transfer frequency (compare pJKI818 and pJKI871, Figure 1D). The 125-bp oriT $T_{\text {SGI1 }}$ sequence proved to be well conserved (95-100\% identity) among the 63 SGI1related IMEs found in public databases as only 11 divergent positions can be found in 17 elements (Figure 2B). These results confirmed that the fully functional ori $T_{\mathrm{SGI}}$ is located between 18016 and 18140 bp of SGI1.

\section{SGI1 Encodes Two Essential Mobilization Proteins Identification of ORFs Required for SGI1 Mobilization}

The mobSI1 region containing 4 annotated ORFs S019, S020, S022, and S021 (latter is encoded on the upper strand) proved to carry all cis- and trans-acting elements that are required for mobilization of SGI1 (pJKI780 in Figure 1C or the mobsGIdonor strain). Mobilization of plasmid pMNI41 from the TG1Nal::SGI1-C $\mathrm{C}^{\Delta \text { oriT }}$ donor strain, where both S021 and S022 are partially deleted due to the $\Delta$ oriT mutation suggested that the proteins encoded by the ORFs S021 and S022 are not required for in trans mobilization initiated at ori $_{\mathrm{SGI}}$. The $\Delta$ oriT mutation removes 40 and 29 amino acid (AA) residues from the C-terminus of the putative S021 and S022 proteins, respectively, which makes improbable that these proteins (if expressed at all) are still functional. To assess their role, independent $\mathrm{KO}$ mutations were generated in the $5^{\prime}$-end of both ORFs in mobsGI containing plasmids. As expected, transfer rates of both mutants were similar to the respective WT plasmid $\left(2.7 \pm 0.54 \times 10^{-3}\right.$ vs. $2.0 \pm 0.32 \times 10^{-3}$ for the S021 frameshift mutant pMSZ957/WT pMSZ949 and $6.1 \pm 2.7 \times 10^{-4}$ vs. $8.0 \pm 1.7 \times 10^{-4}$ for the S022 KO mutant pJKI774/WT pFOL1372) confirming that ORFs S021 and S022 do not encode proteins that are required for in trans mobilization of SGI1.

On the other hand, KO mutations of S019 and S020 had a deleterious effect on SGI1 conjugation, as the transfer frequency of both mutants dropped below $10^{-7}$ like that of the SGI1-C $\mathrm{C}^{\Delta \text { oriT }}$ mutant (Figure 3A). Interestingly, few transconjugant colonies could be obtained with a frequency of 0.9-2.9 $\times 10^{-8}$. Phenotypic and PCR analyses showed that the rare SGI1-C $\mathrm{C}^{\Delta \mathrm{S} 020}$ and SGI1-C $\mathrm{C}^{\Delta \mathrm{S} 019}$ transconjugants carried SGI1 predominantly alone, unlike SGI1-C $\mathrm{C}^{\Delta \text { oriT }}$ transconjugants, where the residual SGI1 transfer was tightly coupled to the conjugation of the helper plasmid. The absence of helper plasmid in the transconjugants suggested that the KO S020 and KO S019 mutants were trans-mobilized, albeit at a very low frequency. Similar result was obtained when the same KO mutations were introduced into the mobsI1 region cloned in a p15A-based plasmid: the mobilization rates of the resulting KO S020 (pJKI737) and KO S019 (pJKI772) plasmids were 4 orders of magnitude lower than that of the WT control (pFOL1372) and were similar to the negative control (pJKI708). Using the mobsGI1-donor strain, WT transfer rates were observed with both $\mathrm{KO}$ mutant plasmids, indicating the effective transcomplementation by the chromosomally integrated mobsGI region (Figure 3B).

\section{Complementation of S020 and S019 KO Mutants}

ORFs S020 and S019 are separated by a single TTG codon (beyond the stop codon of S020). To assess whether the two ORFs are translated into a single, biologically active fusion protein by a read-through mechanism or into two independent proteins, SGI1-C $\mathrm{C}^{\Delta \mathrm{S} 020}$ and SGI1-C $\mathrm{C}^{\Delta \mathrm{S} 019}$ mutants were complemented with plasmids carrying S020 and S019 


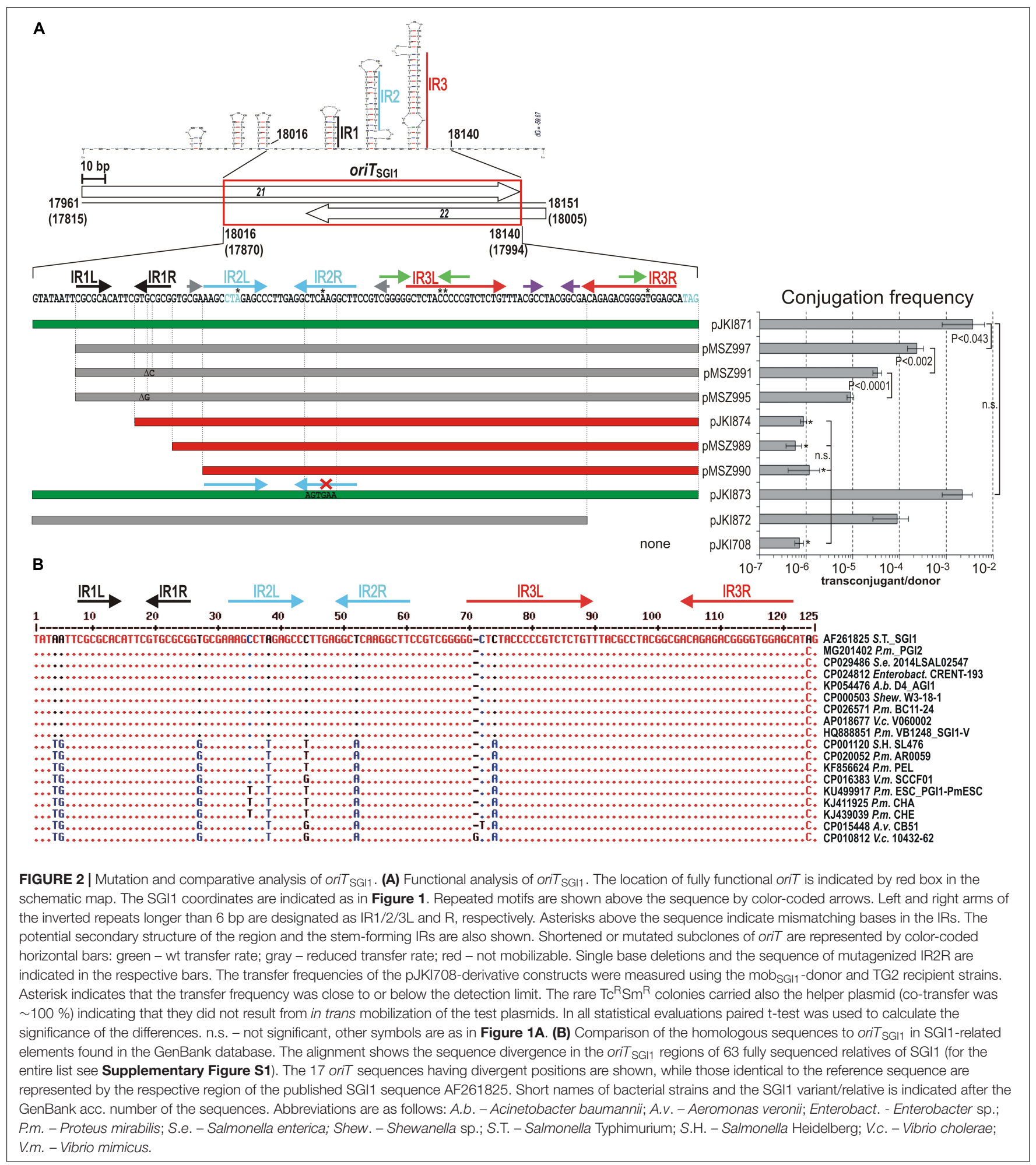

KO mutant mobsI1 regions. While neither SGI1-C mutant could be complemented by the respective $\mathrm{KO}$ mutant mobsGI1 region, SGI1-C $\mathrm{C}^{\Delta \mathrm{S} 020}$ was complemented by S019 $\mathrm{KO}$ mobsGI region and vice versa as efficiently as was complemented by the WT mobsGI region (Figure $3 \mathrm{C}$ ).
This indicated that the two ORFs are translated into independent functional proteins.

Trans complementation of S020 and S019 KO mutant SGI1 was also carried out using expression vectors, which produced the two proteins under the control of $\mathrm{P}_{\text {tac }}$ promoter. These 


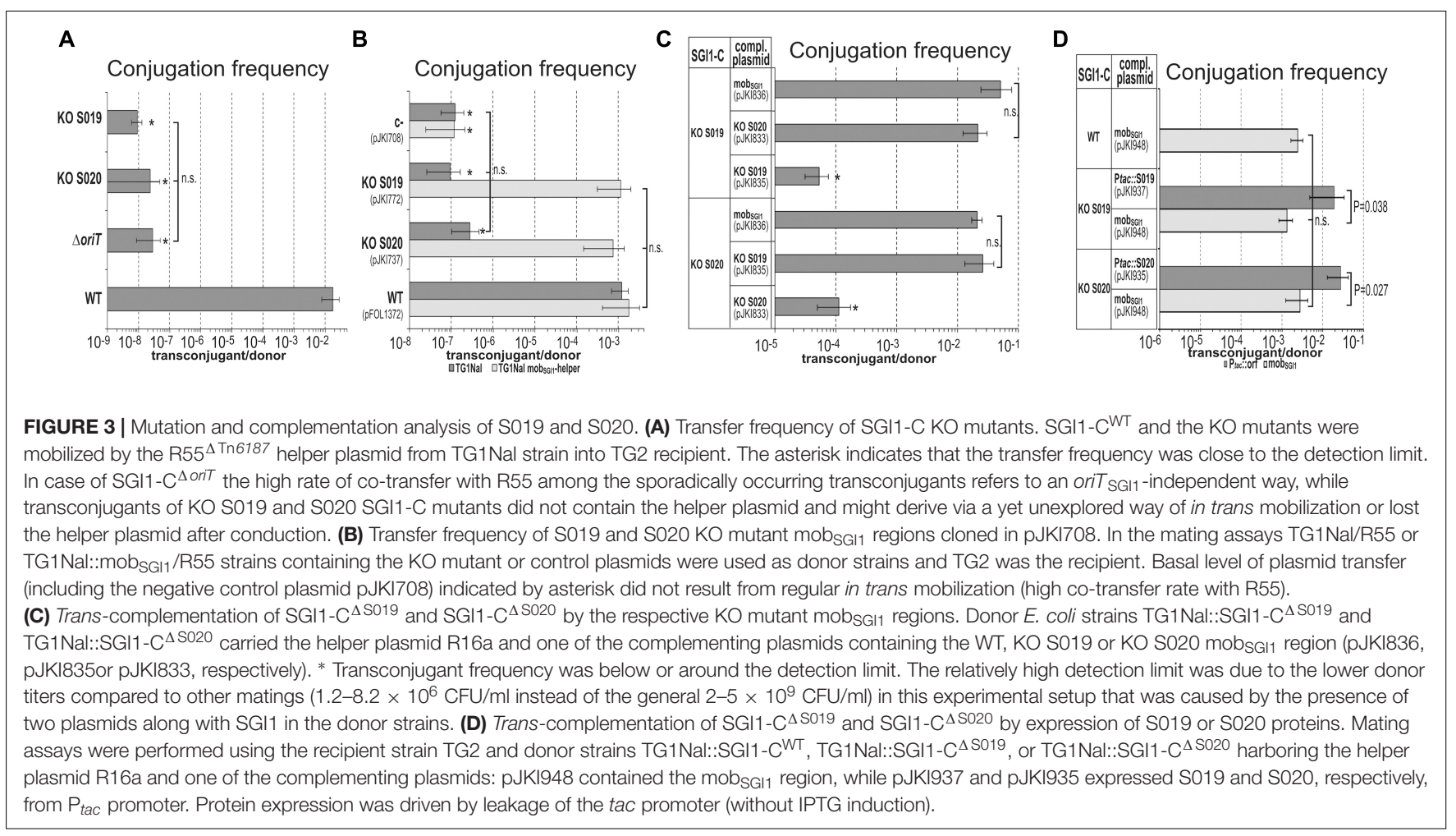

plasmids (pJKI935 and pJKI937 expressing S020 and S019, respectively) were used to complement the TG1Nal::SGI1- $C^{\Delta \text { SO20 }}$ and TG1Nal::SGI1-C $\mathrm{C}^{\Delta \mathrm{S} 019}$ donor strains harboring the IncC helper plasmid R16a. In similar mating assays used previously, both expression plasmids efficiently complemented the respective SGI1 mutants (Figure 3D). About tenfold higher rates of SGI1transfer were observed with the non-mobilizable expression plasmids compared to the mobilizable control pJKI948, probably due to the lack of competition between SGI1 and the complementing plasmids. These data confirmed that ORFs S020 and S019 encode two essential transfer proteins, thus, they have been designated as $m p s A$ and $m p s B$ (mobilization protein of SGI1), respectively.

\section{The Relaxase of IncC Helper Plasmid Is Not Essential for SGI1 Mobilization, but Increases Its Efficiency}

SGI1 exploits the transfer machinery of IncA/C plasmids in multiple manners (Kiss et al., 2015; Carraro et al., 2017), however, the role of the relaxase encoded by the traI gene of IncA/C plasmids in SGI1 mobilization has not yet been analyzed. To assess the possible cooperation between the relaxase and MpsAB proteins in mobilization of SGI1, p15A-based plasmids carrying the WT or mpsA or mpsB KO mutant mobsGI regions were compared in mating assays in the presence of the WT or the relaxase KO mutant helper plasmid, R16a ${ }^{\text {WT }}$ and $R 16 \mathrm{a}^{\Delta \text { TraI }}$, respectively. R16a ${ }^{\mathrm{WT}}$ efficiently mobilized the plasmid containing the WT mobsGI region, while the two KO mutants ( $\triangle m p s A$ and $\triangle m p s B$ ) showed similar transfer rates to the negative control (Figure 4A). These data were in accordance with the previous results obtained with the helper plasmid R55 (Figure 3B) confirming that both $m p s A$ and $m p s B$ genes are required for transfer of the mobsGI region. Interestingly, the helper plasmid R16a ${ }^{\Delta \text { TraI }}$, which is unable to conjugate, mobilized the plasmid containing WT mobsGI region at a frequency of $6.9 \pm 1.4 \times 10^{-6}$, although with ca. 3 logs lower frequency compared to R16a ${ }^{\text {WT }}$ (Figure 4A). Analysis of 10 transconjugants from each independent experiment proved that all were devoid of the helper plasmid and derived from trans-mobilization of the mobsGI plasmid. Moreover, no transconjugant at all was obtained in absence of either $m p s A$ or $m p s B$. The $\mathrm{R}_{16 \mathrm{a}}{ }^{\Delta \mathrm{TraI}}$ was also able to mobilize the chromosomally integrated SGI1-C, although with 5 logs lower efficiency compared to R16a ${ }^{\mathrm{WT}}$ (Figure 4B). Transconjugants were proved to carry SGI1-C integrated at the attB site, and were devoid of the helper plasmid. These results indicate that $m p s A B$ genes are indispensable, while the helper plasmidencoded relaxase is also important for the efficient conjugative transfer initiated at ori $_{\mathrm{SGII}}$. However, mobilization rate of SGI1 remains detectable and significant in absence of the relaxase, suggesting that TraI provides important, but probably different functions in the initiation step of SGI1 transfer from the classical model.

\section{Identification of Promoter Regions Driving the Expression of $m p s A B$ Genes}

Based on the genetic context and the previous results, $m p s A B$ genes were suspected to form a bicistronic operon. To localize the promoter region of this putative operon, the promoter 


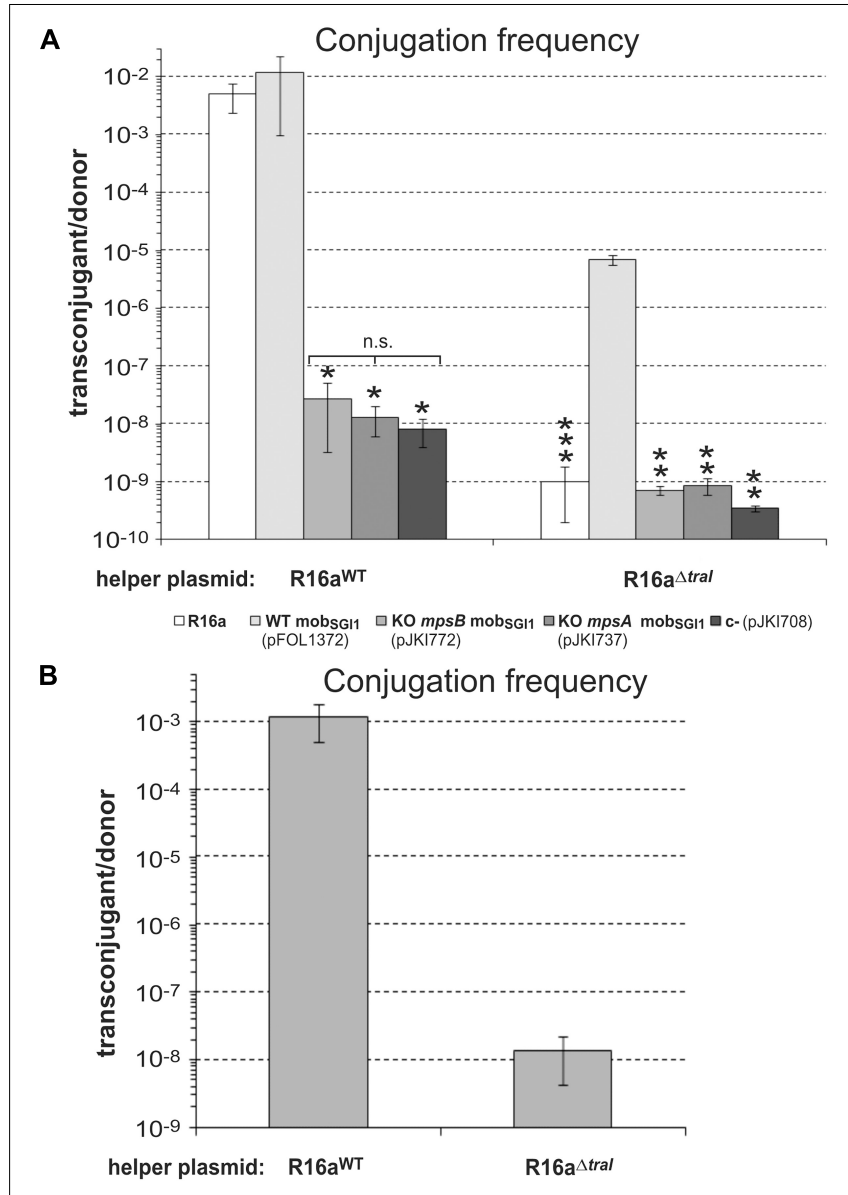

FIGURE 4 | The role of $m p s A, m p s B$ and the helper encoded relaxase tral in SGl1 mobilization. (A) Transfer frequency of the p15A-based plasmids, containing WT (pFOL1372), KO mpsB (KO S019, pJKI772) and KO mpsA (KO S020, pJKI737) mobsGl1 regions was assayed in the presence of the WT or relaxase $\mathrm{KO}$ mutant helper plasmid $\mathrm{R} 16 \mathrm{6}$. For mating, TG1Nal donor and TG90 recipient $E$. coli strains were used. The bars show means of 4 independent experiments except those representing the transfer rate of the helper plasmids. Since the conjugation frequency of the helper plasmids was the same independently of the test plasmids, the transfer rate of the helper plasmids $\mathrm{R} 16 \mathrm{a}^{\mathrm{WT}}$ and $\mathrm{R} 16 \mathrm{a}^{\Delta \text { Tral }}$ correspond to the mean of their transfer frequencies in the 4 different settings that have been repeated 4 times. *Transconjugants indicated by an asterisk carried also the helper plasmid (co-transfer was $\sim 100 \%$ ) suggesting that the transfer of test plasmids did not derive from regular trans-mobilization. **Transfer frequency was below the detection limit (transconjugants were not obtained). ${ }^{* * *}$ Transfer frequency of $\mathrm{R}_{16 \mathrm{a}^{\Delta}}{ }^{\text {Tral }}$ was close to the detection limit, 6 transconjugant colonies were obtained from five independent experiments (mean frequency was $\leq 1.9 \times 10^{-9}$ ). These colonies did not contain test plasmid. (B) Mobilization of SGI1-CWT by the helper plasmids R16a $\mathrm{a}^{\mathrm{WT}}$ and R16a $\mathrm{a}^{\Delta \text { Tral }}$ from TG1Nal::SGl1-C donor into TG90 recipient strain.

activity of sequences located upstream of $m p s A$ was measured in $\beta$-galactosidase assay. Two $\beta$-gal tester plasmids were constructed: the longer region fused to a promoterless lac $Z$ gene extended from the start codon of $m p s A$ to the end of S023 (pMSZ947), while the shorter one extended only to the end of the predicted ORF S022 (pMSZ948). The tester plasmids showed somewhat higher $\beta$-galactosidase activity $(6.4 \pm 1.5$ and
$4.2 \pm 0.5 \mathrm{U}$, respectively) than the negative control plasmid pJKI990 (1.1 $\pm 0.4 \mathrm{U})$ suggesting that a weak promoter could drive the expression of the putative operon [for comparison $\mathrm{P}_{\text {int }}$ of SGI1 produced $\sim 350 \mathrm{U} \beta$-galactosidase in the same assay (Kiss et al., 2015)].

Then, a complementation method was applied to localize this weak promoter more exactly, where the mobilization rate of SGI1-C ${ }^{\triangle m p s A}$ and SGI1-C ${ }^{\Delta m p s B}$ mutants was examined in the presence of R55 ${ }^{\Delta \operatorname{Tn} 6187}$ helper and p15A-based complementing plasmids, which produced MpsA and $\mathrm{MpsB}$ proteins in trans depending on the intactness of the promoter in the cloned mobsGI1 fragments. Both SGI1-C mutants could be complemented to the WT transfer rate achieved with the whole

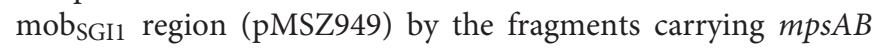
genes and at least the non-coding region between $m p s A$ and S021 (pMSZ976, pMSZ980, Figure 5). This suggested that the $\mathrm{P}_{m p s A B}$ promoter is located in the ca. 100 bp region upstream of the mpsA start codon. Then, primer extension experiments were conducted as previously described (Murányi et al., 2016) to identify the transcriptional start site (TSS) of mpsAB using plasmid pMSZ948. However, in repeated attempts, it failed to detect TSS, which again suggested a low transcription level of $m p s A B$.

Thus, the complementation method was further applied to localize the active promoters by progressively shortening the upstream region of $m p s A$ in the complementing plasmids. Removing of the distal 25 bp (pMSZ984) resulted in a ca. 20fold and 10-fold decrease of the transfer rate of SGI1-C ${ }^{\Delta m p s A}$ and SGI1-C $C^{\Delta m p s B}$, respectively, compared to the shortest fully active plasmid pMSZ976 (Figure 5). The similarly, decreasing complementation efficiency observed with the two KO mutants supported that the two ORFs are expressed from a common promoter located upstream of mpsA. Interestingly, pMSZ993, carrying only $20 \mathrm{bp}$ segment upstream of the $m p s A$ start codon, achieved relatively efficient complementation of SGI1$\mathrm{C}^{\triangle m p s A}$ even though this segment was too short to contain an intact promoter. This raised the possibility that MpsA protein might be translated from the second, in-frame Met codon (17619 bp) instead of the originally identified start codon (17712 bp). This hypothesis was examined using two further complementing plasmids: pMSZ981 contained mpsAB without upstream sequences, while pMSZ996 carried a truncated mpsA beginning with the second Met codon. In both plasmids, the $\operatorname{rrn} B$ T1T2 terminators were inserted immediately upstream of the start codons to prevent any promoter activities from the plasmid backbone. The construct with truncated mpsA (pMSZ996) could not complement SGI1-C $\mathrm{C}^{\triangle m p s A}$, while pMSZ981 containing only the coding sequence of $m p s A B$ without promoter region had a residual activity in complementation of SGI1-C ${ }^{\Delta m p s A}$ (ca. 540 -fold reduction compared to the whole mobSGI region, pMSZ949, Figure 5). This result excluded that translation of MpsA protein starts from the in-frame Met codon and confirmed again that a very low level of MpsA expression is sufficient for complementation.

On the other hand, SGI1-C $C^{\Delta m p s B}$ could be complemented by both constructs at a comparable level observed with pMSZ984, indicating that $m p s B$ can also be expressed independently from 


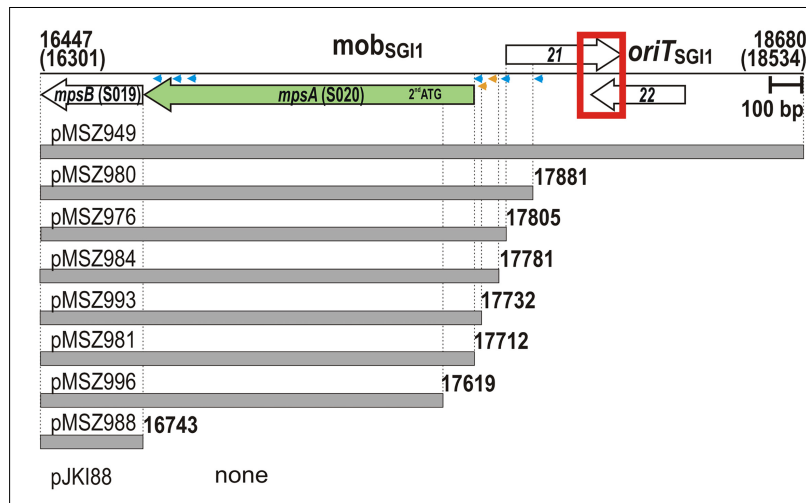
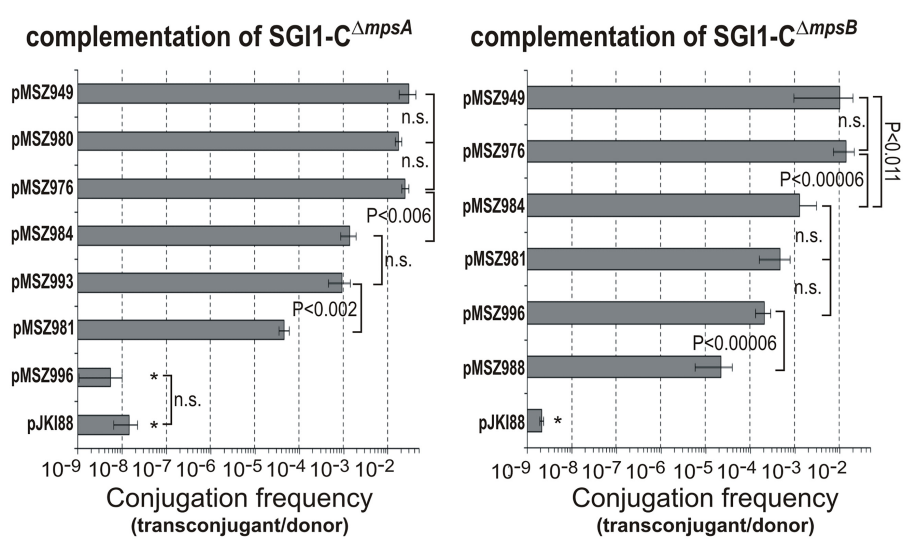

FIGURE 5 | Identification of promoter regions driving expression of $m p s A$ and $m p s B$. The fragments cloned into the complementing plasmids are shown below the schematic map of mobsGl1 region. Putative promoter elements predicted by BPROM or found by manual search are indicated by blue or yellow arrowheads, respectively. Other symbols are as in Figure 1B. Transfer frequency of SGl1-C ${ }^{\Delta S 020}$ and SGI1-C ${ }^{\Delta S 019}$ was measured in the presence of one of the complementing plasmids and the helper plasmid R55 ${ }^{\Delta \text { Tn } 6187}$. Plasmid pMSZ984 showed high st. deviations in three independent complementation assays carried out with $3-6$ replicates with SGI1-C ${ }^{\Delta S 019}$, thus the complementation efficiency of this plasmid could not exactly be determined. However, mean values appeared similar to that of pMSZ981 and pMSZ996. *Transconjugants resulting from oriT SGl1-independent transfer pathway.

$\mathrm{P}_{m p s A B}$. Interestingly, like SGI1-C $\mathrm{C}^{\Delta m p s A}$, the transfer of SGI1$\mathrm{C}^{\Delta m p s B}$ could be complemented by a construct, which contained only the coding sequence of $m p s B$ separated from the plasmid backbone by $\operatorname{rrnB}$ terminators. To detect TSS of $m p s B$, an analogous plasmid to pMSZ948 was constructed, which carried the 227 bp upstream region of $m p s B$ (the $3^{\prime}$ part of $m p s A$ ) fused to the promoterless lacZ gene (pMSZ1017), and primer extension assay was carried out, but without positive result. These suggested again that low level of MpsB can ensure the complementation of SGI1-C $C^{\Delta m p s B}$ (Figure 5). The complementation data indicated that the $m p s A B$ genes can be transcribed into a bicistronic mRNA from a common promoter region $\mathrm{P}_{m p s A B}$, however, $m p s B$ appeared to be expressed also from its own promoter $\mathrm{P}_{m p s B}$ located in the $3^{\prime}$-end of $m p s A$.

The promoter search using BPROM server and additional thorough examination of the sequence revealed several putative promoters in the upstream regions of $m p s A$ and $m p s B$ (Figure 5). Their high divergence from the consensus of $\sigma^{70}$ promoters and the low score values supported our earlier assumption that both the bicistronic $m p s A B$ and $m p s B$ mRNAs are synthesized at a low level from the two promoter regions.

\section{DISCUSSION}

The boom in bacterial genome sequencing in the last decade highly contributed to the discovery of numerous MGIs of the SGI1 and related families gathering IMEs implicated in the spread of antimicrobial resistance among several Gramnegative pathogens such as S. enterica serovars, Proteus mirabilis strains and a few other species. Despite the evidence that SGI1-related elements are specifically mobilized by conjugative IncA/C plasmids, their own mobilization components such as oriT and self-encoded conjugation proteins have not yet been identified.
In this study, the essential SGI1 conjugative mobilization functions were found to be clustered in the $2.2-\mathrm{kb}_{\text {mob }}$ (I1 region (Figures 1C,D) carrying the ori $T_{\mathrm{SGI}}$ and two essential genes (ORFs S020 and S019) named $m p s A$ and $m p s B$. The fully active minimal oriT sequence has been localized in the vicinity of the $m p s A B$ genes between 18016 and 18140 bp positions (GenBank accession AF261825), which includes the overlapping $3^{\prime}$ parts of two small predicted ORFs S021 and S022. Like many oriT regions identified to date, the ori $T_{\mathrm{SGI}}$ also contains inverted repeat motifs. Three long IR motifs ( $\geq 7 \mathrm{bp}$, IRs1-3) have been found in the 125-bp minimal ori $T_{\mathrm{SGI} 1}$. The most important one is the GC-rich 7-bp perfect IR1 (Figure 2A), suggesting its functional implication in binding relaxosome proteins and that the potential nic-site is probably located near this motif. Different methodologies have been successfully applied to identify nic-sites of several well-known conjugative plasmids and ICEs (Pansegrau et al., 1993; Llosa et al., 1995; Zechner et al., 1997; Núñez and De La Cruz, 2001; Varsaki et al., 2003; Lee and Grossman, 2007; Chen et al., 2010; Tsvetkova et al., 2010; Grove et al., 2013). However, the nic-site of one of the best studied ICEs, SXT, and the related IncA/C plasmids could not be defined yet [V. Burrus personal communication, (Hegyi et al., 2017)]. Although many efforts have been made to identify the nic-site in ori $T_{\text {SGI1 }}$ applying biochemical (primer extension, RACE PCR, in vitro nicking assay) and in vivo (interrupted mating) methods, all failed to give convincing results to date (data not shown).

In contrast to most ori $T$ regions, ori $T_{\mathrm{SGI}}$ is a GC-rich $(60 \%)$ sequence compared to the SGI1 backbone (44\%). Similar high GC-content (61\%) can be seen in both oriTs of ICEclc element (Miyazaki and Van Der Meer, 2011), however, in this case the entire ICE has also high (62.5\%) GC-content.

The largest part or the entire mobsGI region is present in all the 63 fully sequenced SGI1-related IMEs (Supplementary Figure S1). In the distant relatives (see bottom of Supplementary Figure S1), the $3^{\prime}$-end of $m p s B$ homologs are more divergent 
and the distal part (ca. $210 \mathrm{bp}$ at the $3^{\prime}$-end of mobsGI region) of the non-coding region adjacent to S023 along with ORF S023 are missing (e.g., in AGI1 or PGI2). The nucleotide identity of the mobsGI-homolog regions to the corresponding SGI1 reference varies from 85 to $100 \%$. The 125 bp ori $T_{\text {SGI1 }}$ sequence is highly conserved (95-100\% identity) among the SGI1-related IMEs as only 11 divergent positions can be found in 17 out of the 63 elements (Figure 2B). Four and three divergent positions occur in the IR2Lspace-IR2R region and in the $5^{\prime}$-end of IR3L, respectively, while the GC-rich 7-bp perfect IR1 is fully conserved. These observations are congruent with the conclusion of the experimental results, strengthening the importance of this region in SGI1 mobilization.

OriT $_{\mathrm{SGI}}$ does not show striking similarity to that of IncA/C plasmids (ori $T_{\mathrm{A} / \mathrm{C}}$ ), either in genetic context, sequence motifs or potential secondary structures (Supplementary Figures S2A,B). Ori $_{\mathrm{A} / \mathrm{C}}$ locates in an intergenic region between divergent genes and overlaps the promoter of one of them, mobI, which encodes a plasmid-specific transfer factor that is indispensable for conjugation of IncA/C plasmids, but not required for SGI1 mobilization (Hegyi et al., 2017). Although ori $T_{\mathrm{A} / \mathrm{C}}$ also contains several IRs, these are shorter and share no sequence similarity to those of oriT $T_{\mathrm{SGI}}$. Furthermore, the most important part of ori $\mathrm{T}_{\mathrm{A} / \mathrm{C}}$ is a 14 -bp direct repeat motif, which has no similar counterparts in ori $T_{\mathrm{SGI}}$. Other striking difference is the GC-content: while oriT $T_{\mathrm{SGI}}$ is rather a GCrich sequence, ori $T_{\mathrm{A} / \mathrm{C}}$ has a low GC content (37.4\%). The only similarity with ori $_{\mathrm{A} / \mathrm{C}}$ as well as with oriTs of many other mobilizable elements is the location of ori $T_{\mathrm{SGI}}$ in the vicinity of mobilization genes.

Two pivotal SGI1-encoded transfer genes, ORFs S020 and S019, have been identified and were renamed here as $m p s A$ and $m p s B$, respectively. Unlike $m p s A B$ genes, the two other small predicted ORFs of mobsi1, S021 and S022, do not appear to encode proteins that are involved in transfer process. Complementation experiments showed that $m p s A$ and $m p s B$ separated by a single codon beyond the stop codon of $m s p A$ are translated into independent polypeptides, in part, from a bicistronic mRNA, however, MpsB protein can also be expressed from its own mRNA (Figures 3C,D, 5). Interestingly, the sequential reduction of the upstream region of $m p s A$ caused gradual decrease in complementation efficiencies of the respective $\mathrm{KO}$ mutant, suggesting the presence of at least two different functional promoters in this region. The sequence-based prediction, which identified several putative promoters in the intergenic region between mpsA and S021 and in the $5^{\prime}$ part of S021 (Figure 5) supported this assumption. By BPROM prediction three potential promoters located upstream of $m p s B$ (in the $3^{\prime}$ part of $m p s A$ ) were found approving that $m p s B$ has also its own promoter region. All of these promoter-like sequences show low similarity to the $\sigma^{70}$ consensus. The weak promoter activity observed in $\beta$-gal assays, the negative results of primer extension assays to detect TSSs and the fact that plasmid constructs carrying $m p s B$ or $m p s A B$ coding sequences without their upstream regions could complement the respective
KO mutants indicated that very low amount of MpsA and MpsB proteins are sufficient to reach detectable or even WT transfer rates.

Despite the crucial role of oriT $T_{\mathrm{SGI} 1}, \mathrm{MpsA}$ and $\mathrm{MpsB}$ proteins in SGI1 mobilization, a very low transfer frequency was observed with each SGI1-C KO mutants (around $10^{-8}$ /donor, see Figures $\mathbf{3 A}, \mathbf{4 A}$ ) and mobsGI1-plasmids (Figure 3B) suggesting that the helper plasmid is somehow able to mobilize SGI1 at a very low level independently of cis- and trans-acting mobilization factors of SGI1. In case of deletion of oriT $T_{\text {SGI1 }}$, the majority $(\geq 97 \%)$ of the rare transconjugants contained also the helper plasmid suggesting that the transfer of SGI1 or the test plasmids was not independent of the transfer of the helper plasmid. The most plausible explanation for this phenomenon is that co-integrates was formed with the helper plasmid. The low transfer rate and the lack of extensive homology between SGI1-C derivatives or mobsGI1-plasmids and the helper plasmids suggest the involvement of a recA-independent illegitimate recombination process. Further support for a recA-independent mechanism may be that similar transfer rates were observed with the ori $T_{\mathrm{SGI1}}$-free pJKI708 and its derivatives when the $\mathrm{rec}^{-}$E. coli TG2 was used as donor strain (Figure 1C). A co-integrate based transfer mechanism has been reported previously (Hegyi et al., 2017), however, in that case the high transfer frequency was the consequence of an efficient site-specific recombination. The sporadically occurring helper-free transconjugants obtained with $m p s A$ or $m p s B \mathrm{KO}$ mobsGI1-plasmids $(\leq 3 \%$, Figures $3 \mathbf{B}, 4 \mathbf{A})$ and the generally helper-free SGI1-C ${ }^{\Delta m p s A}$ or SGI1-C $\mathrm{C}^{\Delta m p s B}$ transconjugants (Figure 3A) may represent a yet unexplored way of inefficient mobilization by IncA/C plasmids in absence of the major transfer proteins MpsA and MpsB.

Both the homology searches and the structure predictions advocate that MpsA protein belongs to the Tyrrecombinase/integrase superfamily of DNA breaking-rejoining enzymes. The 126-225 AA tract of MpsA shows high homology to the DNA_BRE_C conserved C-terminal catalytic domain that is characteristic of site-specific integrases, bacterial recombinases XerD/C and type IB topoisomerases.

Phyre 2 modeling confirmed that MpsA is related to the Tyr-recombinase family, as the best templates with known 3D-structure were XerH (Fold library id.: c5jjvA) and Cre recombinase (Fold library id.: c1ma7A) with $15-13 \%$ sequence identity, $91-94 \%$ coverage and 100\% confidence. All the other hits showing $>90 \%$ coverage were also site-specific Tyrrecombinases (XerC,D; phage integrases). Similar result was obtained from Swiss-Model. Based on AA sequence alignments with related recombinases, the conserved catalytic nucleophile tyrosine residue was predicted at the C-terminus of MpsA protein (Y319). In addition to the Y319, DELTA-BLAST allowed to identify the R162, H247, R250, H251 in MpsA matching with the catalytic residues of the active sites of bacteriophage $\mathrm{Hp} 1$ Integrase (1AIH_A), Cre (2CRX_B), $\lambda$ Integrase (1AE9_A) and IntI4 (2A3V_A). The mpsA gene appears to be well conserved among the 63 SGI1-related elements as only 19 show some sequence divergence compared 
to the reference SGI1 (99-87\% identity). Although these base variations cause several changes in the predicted amino acid sequences (Supplementary Figure S3A), the only case when MpsA protein is apparently truncated and possibly inactive is the Aeromonas veronii CB51.

The $m p s B$ gene encoding for the other essential mobilization factor of SGI1 is also similarly, conserved as only 17 of 63 elements show some DNA sequence variations in this gene (99-76\% identity). These changes cause AA substitutions at 18 positions of the predicted MpsB proteins, however, truncation of the protein occurs only in $V$. mimicus SCCF01 and incomplete protein can be expressed in A. veronii CB51 (Supplementary Figure S3B). Using MotifFinder, the 98-AA-long MpsB protein was predicted to contain a phage integrase N-terminal SAM4 like domain (PF13495, Pfam database), which was also supported by the best Phyre 2 modeling based on the lambda integrase-like $\mathrm{N}$-terminal domain template (Fold library id.: d1f44a1) and by Swiss-model where, following two eukaryotic proteins spectrin and plectin, the best hits were the N-terminal domains of XerH and Cre. Based on these data and PSIPRED secondary structure prediction (Buchan et al., 2013), MpsB appears to be an independent domain-like protein containing $4 \alpha$-helices of which the last three (20-82 AA) are related to a portion of the $\mathrm{N}$-terminal core binding domain of lambda integrases (Swalla et al., 2003). This raises the possibility that $\mathrm{MpsB}$ is involved in DNA binding at ori $T_{\mathrm{SGI}}$, however, a protein-recruiting function into the initiation complex cannot be ruled out either.

SGI1 exploits the transfer machinery of IncA/C plasmids in multiple manners (Kiss et al., 2015; Carraro et al., 2017), however, the role of the plasmid-borne relaxase TraI in SGI1 mobilization has not yet been analyzed. The mobilization assays using $\mathrm{R} 16^{\Delta \mathrm{TraI}}$ helper plasmid provided an unexpected result. While conjugation of the helper plasmid appears strictly TraIdependent, the WT mobsGI1-plasmid and SGI1-C proved to be mobilizable in absence of TraI (Figure 4), albeit with orders of magnitude less efficiently. Considering the striking difference between ori $T_{\mathrm{SGI}}$ and ori $_{\mathrm{A} / \mathrm{C}}$ (Supplementary Figure S2), it is unlikely that similar relaxosome complexes are formed at these oriTs. The fact that SGI1 mobilization was found to be possible in absence of the helper-encoded relaxase suggests that other SGI1encoded proteins ensure the essential relaxase functions, at least in part, in the SGI1 relaxosome at ori $T_{\mathrm{SGI}}$.

The IncA/C relaxase TraI, although significantly increases the SGI1 transfer by a yet unknown mechanism, seems unable to mobilize oriT $T_{\mathrm{SGI}}$ in absence of MpsA or MpsB. Its role might be the unwinding of donor DNA or delivery of the relaxosome to T4SS by recruiting the coupling protein. Since the components of the initiation complex of IncA/C plasmids are only predictable through their homologies to those of wellstudied model systems, the functions of TraI and involvement of other IncA/C-encoded proteins in the plasmid transfer and SGI1 mobilization need further investigations. In addition to the DNA nicking domain, other functional domains of relaxases, such as helicase domains (Llosa et al., 1996; Cheng et al., 2011; Alperi et al., 2013) and translocation signals, which are required for binding by the cognate coupling proteins and delivery of the relaxase-DNA complex to the T4SS (Lang et al., 2010), have been identified in several conjugation systems. Since the helicase domain seems to be the peculiarity of the IncW/N/F family relaxases (Llosa et al., 1996) and such domain have not yet been identified in TraI of IncA/C plasmids, the most possible way by which TraI can enhance SGI1 mobilization is to facilitate the transport of the relaxosome complex to or even through the T4SS. In addition to TraI, some conjugation systems require several auxiliary relaxosome components. In the case of IncP $\alpha$ plasmid $\mathrm{RP} 4$, TraJ protein is responsible for sequence-specific recognition of oriT and directing TraI to the origin of transfer (Ziegelin et al., 1989), TraH stabilizes the oriT-DNA complex through specific protein-protein interactions (Pansegrau et al., 1990) and TraK stimulates the relaxation by wrapping DNA near oriT (Ziegelin et al., 1992). In IncW plasmid R388, TrwA binds specifically to oriT and stimulates the ATPase activity of the coupling protein TrwB, which is indispensable to link the relaxosome complex to T4SS (Cabezón et al., 2015). Similar auxiliary factors are not yet known in the conjugative apparatus of IncA/C plasmids. Besides the structural components and the assembly factors of T4SS, only TraI and the indispensable transfer factor MobI have been described in IncA/C transfer. Although the exact function of MobI is not clear, it appears not to be involved in SGI1 mobilization (Hegyi et al., 2017).

Based on the results presented here we suggest that MpsA functions as an atypical relaxase of SGI1, which needs MpsB for binding and/or nicking ori $T_{\mathrm{SGI}}$, but does not require TraI for the initial step of SGI1 relaxosome formation. This explanation is strengthened by the recent description of the atypical relaxase TcpM of plasmid pCW3 from Clostridium perfringens that appears related to tyrosine recombinases (our Phyre2 modeling showed the best homology of TcpM with XerH) without sequence similarity to known relaxases (Wisniewski et al., 2016). The fact that $m p s B \mathrm{KO}$ mutant mobsGI1-plasmid could not be mobilized even by a WT helper plasmid (Figure 4) suggests that MpsB rather has a role in the initiation of transfer (maybe in binding ori $T_{\text {SGI1 }}$ together with MpsA), than recruiting additional proteins like TraI. The relaxase TraI might be involved in unwinding the donor DNA or more possibly in the transport of the relaxosome to T4SS. The key elements and an accessory partner of the SGI1 relaxosome have been here identified, however, the understanding their exact role in the conjugative DNA processes needs further investigations.

\section{DATA AVAILABILITY}

All datasets generated for this study are included in the manuscript and/or the Supplementary Files.

\section{AUTHOR CONTRIBUTIONS}

JK, BD, AC, and FO conceived the project. JK, MS, AH, GD, $\mathrm{KP}, \mathrm{BD}, \mathrm{IN}$, and $\mathrm{FO}$ designed and carried out the experiments and analyzed the data. JK and BD performed the bioinformatic analyses, JK prepared the figures. JK, BD, AC, and $\mathrm{AH}$ wrote the manuscript. All the authors reviewed the manuscript. 


\section{FUNDING}

This work was supported by the Hungarian Scientific Research Fund K 105635 and NKFI K 128203 to JK and by public funds from the French National Institute of Agricultural Research to BD. HA was supported by a fellowship of Campus France. GD was supported by a Ph.D. fellowship from Ministère de l'Enseignement Supérieur et de la Recherche, France. IN was supported by the Ph.D. fellowship of Eötvös Loránd University, Budapest, Hungary.

\section{REFERENCES}

Alperi, A., Larrea, D., Fernández-González, E., Dehio, C., Zechner, E. L., and Llosa, M. (2013). A translocation motif in relaxase trwc specifically affects recruitment by its conjugative type IV secretion system. J. Bacteriol. 195, 4999-5006. doi: 10.1128/JB.00367-13

Ambrose, S. J., Harmer, C. J., and Hall, R. M. (2018). Compatibility and entry exclusion of IncA and IncC plasmids revisited: IncA and IncC plasmids are compatible. Plasmid 9, 7-12. doi: 10.1016/j.plasmid.2018.02.002

Arnold, K., Bordoli, L., Kopp, J., and Schwede, T. (2006). The SWISSMODEL workspace: a web-based environment for protein structure homology modelling. Bioinformatics 22, 195-201. doi: 10.1093/bioinformatics/bti770

Avila, P., Núñez, B., and de la Cruz, F. (1996). Plasmid R6K contains two functional oriTs which can assemble simultaneously in relaxosomes in vivo. J. Mol. Biol. 261, 135-143. doi: 10.1006/jmbi.1996.0447

Bellanger, X., Payot, S., Leblond-Bourget, N., and Guédon, G. (2014). Conjugative and mobilizable genomic islands in bacteria: evolution and diversity. FEMS Microbiol. Rev. 38, 720-760. doi: 10.1111/1574-6976.12058

Boyd, D., Peters, G. A., Cloeckaert, A., Boumedine, K. S., Chaslus-Dancla, E., Imberechts, H., et al. (2001). Complete nucleotide sequence of a 43-kilobase genomic island associated with the multidrug resistance region of Salmonella enterica serovar Typhimurium DT104 and its identification in phage type DT120 and serovar Agona. J. Bacteriol. 183, 5725-5732. doi: 10.1128/JB.183. 19.5725-5732.2001

Boyd, D. A., Peters, G. A., Ng, L., and Mulvey, M. R. (2000). Partial characterization of a genomic island associated with the multidrug resistance region of Salmonella enterica Typhymurium. FEMS Microbiol. Lett. 189, 285-291. doi: 10.1111/j.1574-6968.2000.tb09245.x

Buchan, D. W. A., Minneci, F., Nugent, T. C. O., Bryson, K., and Jones, D. T. (2013). Scalable web services for the PSIPRED protein analysis workbench. Nucleic Acids Res. 41, W349-W357. doi: 10.1093/nar/gkt381

Cabezón, E., Ripoll-Rozada, J., Peña, A., de la Cruz, F., and Arechaga, I. (2015). Towards an integrated model of bacterial conjugation. FEMS Microbiol. Rev. 39, 81-95. doi: 10.1111/1574-6976.12085

Carraro, N., and Burrus, V. (2014). Biology of three ICE families: SXT/R391, ICEBs1, and ICESt1/ICESt3. Microbiol. Spectr. 2, 1-20. doi: 10.1128/ microbiolspec.MDNA3-0008-2014

Carraro, N., Durand, R., Rivard, N., Anquetil, C., Barrette, C., Humbert, M., et al. (2017). Salmonella genomic island 1 (SGI1) reshapes the mating apparatus of IncC conjugative plasmids to promote self-propagation. PLoS Genet. 13:e1006705. doi: 10.1371/journal.pgen.1006705

Carraro, N., Matteau, D., Luo, P., Rodrigue, S., and Burrus, V. (2014). The master activator of IncA/C conjugative plasmids stimulates genomic islands and multidrug resistance dissemination. PLoS Genet. 10:e1004714. doi: 10.1371/ journal.pgen.1004714

Carraro, N., Rivard, N., Ceccarelli, D., Colwell, R. R., and Burrus, V. (2016). IncA/C conjugative plasmids mobilize a new family of multidrug resistance islands in clinical Vibrio cholerae non-O1/non-O139 isolates from Haiti. mBio 7:e00509-16. doi: 10.1128/mBio.00509-16

Ceccarelli, D., Daccord, A., René, M., and Burrus, V. (2008). Identification of the origin of transfer (oriT) and a new gene required for mobilization of the SXT/R391 family of integrating conjugative elements. J. Bacteriol. 190, 5328-5338. doi: 10.1128/JB.00150-08

\section{ACKNOWLEDGMENTS}

We thank Erika Sztánáné-Keresztúri and Mária Turai for the excellent technical assistance.

\section{SUPPLEMENTARY MATERIAL}

The Supplementary Material for this article can be found online at: https://www.frontiersin.org/articles/10.3389/fmicb. 2019.00457/full\#supplementary-material

Chen, J., Pappas, D. L., and Galli, D. M. (2010). Mapping of the nick site on conjugative plasmid pVT745 by interrupted mating. Plasmid 63, 136-142. doi: 10.1016/j.plasmid.2010.02.001

Cheng, Y., McNamara, D. E., Miley, M. J., Nash, R. P., and Redinbo, M. R. (2011). Functional characterization of the multidomain $\mathrm{F}$ plasmid TraI relaxasehelicase. J. Biol. Chem. 286, 12670-12682. doi: 10.1074/jbc.M110.207563

Corpet, F. (1988). Multiple sequence alignment with hierarchical clustering. Nucleic Acids Res. 16, 10881-10890. doi: 10.1093/nar/16.22.10881

Daccord, A., Ceccarelli, D., and Burrus, V. (2010). Integrating conjugative elements of the SXT/R391 family trigger the excision and drive the mobilization of a new class of Vibrio genomic islands. Mol. Microbiol. 78, 576-588. doi: 10.1111/ j.1365-2958.2010.07364.x

Daccord, A., Ceccarelli, D., Rodrigue, S., and Burrus, V. (2013). Comparative analysis of mobilizable genomic islands. J. Bacteriol. 195, 606-614. doi: 10.1128/ JB.01985-12

Datsenko, K. A., and Wanner, B. L. (2000). One-step inactivation of chromosomal genes in Escherichia coli K-12 using PCR products. Proc. Natl. Acad. Sci. U.S.A. 97, 6640-6645. doi: 10.1073/pnas.120163297

Datta, N., and Hedges, R. W. (1973). R factors of compatibility group A. J. Gen. Microbiol. 74, 335-337. doi: 10.1099/00221287-74-2-335

De La Cruz, F., Frost, L. S., Meyer, R. J., and Zechner, E. L. (2010). Conjugative DNA metabolism in Gram-negative bacteria. FEMS Microbiol. Rev. 34, 18-40. doi: 10.1111/j.1574-6976.2009.00195.x

Douard, G., Praud, K., Cloeckaert, A., and Doublet, B. (2010). The Salmonella genomic island 1 is specifically mobilized in trans by the $\mathrm{IncA} / \mathrm{C}$ multidrug resistance plasmid family. PLoS One 5:e15302. doi: 10.1371/journal.pone. 0015302

Doublet, B., Boyd, D., Mulvey, M. R., and Cloeckaert, A. (2005). The Salmonella genomic island 1 is an integrative mobilizable element. Mol. Microbiol. 55, 1911-1924. doi: 10.1111/j.1365-2958.2005.04520.x

Doublet, B., Praud, K., Bertrand, S., Collard, J.-M., Weill, F.-X., and Cloeckaert, A. (2008). Novel insertion sequence- and transposonmediated genetic rearrangements in genomic island SGI1 of Salmonella enterica serovar Kentucky. Antimicrob. Agents Chemother. 52, 3745-3754. doi: 10.1128/AAC.00525-08

Francia, M. V., and Clewell, D. B. (2002). Transfer origins in the conjugative Enterococcus faecalis plasmids pAD1 and pAM373: identification of the pAD1 nic site, a specific relaxase and a possible TraG-like protein. Mol. Microbiol. 45, 375-395. doi: 10.1046/j.1365-2958.2002.03007.x

Francia, M. V., Haas, W., Wirth, R., Samberger, E., Muscholl-Silberhorn, A., Gilmore, M. S., et al. (2001). Completion of the nucleotide sequence of the Enterococcus faecalis conjugative virulence plasmid pAD1 and identification of a second transfer origin. Plasmid 46, 117-127. doi: 10.1006/plas.2001.1533

Frost, L. S., Ippen-Ihler, K., and Skurray, R. A. (1994). Analysis of the sequence and gene products of the transfer region of the F sex factor. Microbiol. Rev. 5, $162-210$.

Fürste, J. P., Pansegrau, W., Ziegelin, G., Kröger, M., and Lanka, E. (1989). Conjugative transfer of promiscuous IncP plasmids: interaction of plasmidencoded products with the transfer origin. Proc. Natl. Acad. Sci. U.S.A. 86, 1771-1775. doi: 10.1073/pnas.86.6.1771

Garcillán-Barcia, M. P., Francia, M. V., and De La Cruz, F. (2009). The diversity of conjugative relaxases and its application in plasmid classification. FEMS Microbiol. Rev. 33, 657-687. doi: 10.1111/j.1574-6976.2009.00168.x 
Grove, J. I., Alandiyjany, M. N., and Delahay, R. M. (2013). Site-specific relaxase activity of a VirD2-like protein encoded within the $\mathrm{tfs} 4$ genomic island of helicobacter pylori. J. Biol. Chem. 288, 26385-26396. doi: 10.1074/jbc.M113. 496430

Hamidian, M., Holt, K. E., and Hall, R. M. (2015). Genomic resistance island AGI1 carrying a complex class 1 integron in a multiply antibiotic-resistant ST25 Acinetobacter baumannii isolate. J. Antimicrob. Chemother. 70, 2519-2523. doi: $10.1093 / \mathrm{jac} / \mathrm{dkv} 137$

Harmer, C. J., Hamidian, M., Ambrose, S. J., and Hall, R. M. (2016). Destabilization of IncA and IncC plasmids by SGI1 and SGI2 type Salmonella genomic islands. Plasmid 8, 51-57. doi: 10.1016/j.plasmid.2016.09.003

Hegyi, A., Szabó, M., Olasz, F., and Kiss, J. (2017). Identification of oriT and a recombination hot spot in the IncA/C plasmid backbone. Sci. Rep. 7:10595. doi: 10.1038/s41598-017-11097-0

Herrero, M., De Lorenzo, V., and Timmis, K. N. (1990). Transposon vectors containing non-antibiotic resistance selection markers for cloning and stable chromosomal insertion of foreign genes in gram-negative bacteria. J. Bacteriol. 172, 6557-6567. doi: 10.1128/jb.172.11.6557-6567.1990

Huguet, K. T., Gonnet, M., Doublet, B., and Cloeckaert, A. (2016). A toxin antitoxin system promotes the maintenance of the IncA/C-mobilizable Salmonella genomic Island 1. Sci. Rep. 6:32285. doi: 10.1038/srep32285

Jaworski, D. D., and Clewell, D. B. (1995). A functional origin of transfer (oriT) on the conjugative transposon Tn916. J. Bacteriol. 177, 6644-6651. doi: 10.1128/jb. 177.22.6644-6651.1995

Johnson, S. L., Khiani, A., Bishop-Lilly, K. A., Chapman, C., Patel, M., Verratti, K., et al. (2015). Complete genome assemblies for two single-chromosome Vibrio cholerae isolates, strains 1154-74 (Serogroup O49) and 10432-62 (Serogroup O27). Genome Announc. 3:e00462-15. doi: 10.1128/genomeA. 00462-15

Kelley, L. A., Mezulis, S., Yates, C. M., Wass, M. N., and Sternberg, M. J. E. (2015). The Phyre2 web portal for protein modeling, prediction and analysis. Nat. Protoc. 10, 845-858. doi: 10.1038/nprot.2015.053

Kiss, J., Nagy, B., and Olasz, F. (2012). Stability, entrapment and variant formation of Salmonella genomic island 1. PLoS One 7:e32497. doi: 10.1371/journal.pone. 0032497

Kiss, J., Papp, P. P., Szabó, M., Farkas, T., Murányi, G., Szakállas, E., et al. (2015). The master regulator of IncA/C plasmids is recognized by the Salmonella genomic Island SGI1 as a signal for excision and conjugal transfer. Nucleic Acids Res. 43, 8735-8745. doi: 10.1093/nar/gkv758

Kolisnychenko, V., Plunkett, G., Herring, C. D., Fehér, T., Pósfai, J., Blattner, F. R., et al. (2002). Engineering a reduced Escherichia coli genome. Genome Res. 12, 640-647. doi: 10.1101/gr.217202

Lang, S., Gruber, K., Mihajlovic, S., Arnold, R., Gruber, C. J., Steinlechner, S., et al. (2010). Molecular recognition determinants for type IV secretion of diverse families of conjugative relaxases. Mol. Microbiol. 78, 1539-1555. doi: 10.1111/j. 1365-2958.2010.07423.x

Lee, C. A., and Grossman, A. D. (2007). Identification of the origin of transfer (oriT) and DNA relaxase required for conjugation of the integrative and conjugative element ICEBs1 of Bacillus subtilis. J. Bacteriol. 189, 7254-7261. doi: 10.1128/JB.00932-07

Levings, R. S., Djordjevic, S. P., and Hall, R. M. (2008). SGI2, a relative of Salmonella genomic island SGI1 with an independent origin. Antimicrob. Agents Chemother. 52, 2529-2537. doi: 10.1128/AAC.00189-08

Levings, R. S., Lightfoot, D., Partridge, S. R., Hall, R. M., and Djordjevic, S. P. (2005). The genomic Island SGI1, containing the multiple antibiotic resistance region of Salmonella enterica serovar Typhimurium DT104 or variants of it, is widely distributed in other S. enterica serovars. J. Bacteriol. 187, 4401-4409. doi: 10.1128/JB.187.13.4401

Li, X., Xie, Y., Liu, M., Tai, C., Sun, J., Deng, Z., et al. (2018). OriTfinder: a webbased tool for the identification of origin of transfers in DNA sequences of bacterial mobile genetic elements. Nucleic Acids Res. 46, W229-W234. doi: $10.1093 / \mathrm{nar} / \mathrm{gky} 352$

Liu, M., Li, X., Xie, Y., Bi, D., Sun, J., Li, J., et al. (2019). ICEberg 2.0: an updated database of bacterial integrative and conjugative elements. Nucleic Acids Res. 47, D660-D665. doi: 10.1093/nar/gky1123

Llosa, M., and Alkorta, I. (2017). Coupling proteins in type IV secretion. Curr. Top. Microbiol. Immunol. 413, 143-168. doi: 10.1007/978-3-319-75241-9_6
Llosa, M., Grandoso, G., and de la Cruz, F. (1995). Nicking activity of TrwC directed against the origin of transfer of the IncW plasmid R388. J. Mol. Biol. 246, 54-62. doi: 10.1006/jmbi.1994.0065

Llosa, M., Grandoso, G., Hernando, M. A., and De La Cruz, F. (1996). Functional domains in protein TrwC of plasmid R388: dissected DNA strand transferase and DNA helicase activities reconstitute protein function. J. Mol. Biol. 264, 56-67. doi: 10.1006/jmbi.1996.0623

Miller, J. H. (1972). Experiments in Molecular Genetics. New York, NY: Cold Spring Harbor Laboratory Press.

Miyazaki, R., and Van Der Meer, J. R. (2011). A dual functional origin of transfer in the ICEclc genomic island of Pseudomonas knackmussii B13. Mol. Microbiol. 79, 743-758. doi: 10.1111/j.1365-2958.2010.07484.x

Murányi, G., Szabó, M., Olasz, F., and Kiss, J. (2016). Determination and analysis of the putative AcaCD-responsive promoters of Salmonella genomic island 1. PLoS One 11:e0164561. doi: 10.1371/journal.pone.0164561

Núñez, B., and De La Cruz, F. (2001). Two atypical mobilization proteins are involved in plasmid CloDF13 relaxation. Mol. Microbiol. 39, 1088-1099. doi: 10.1046/j.1365-2958.2001.02308.x

Pansegrau, W., Balzer, D., Kruft, V., Lurz, R., and Lanka, E. (1990). In vitro assembly of relaxosomes at the transfer origin of plasmid RP4. Proc. Natl. Acad. Sci. U.S.A. 87, 6555-6559. doi: 10.1073/pnas.87.17.6555

Pansegrau, W., and Lanka, E. (1991). Common sequence motifs in DNA relaxases and nick regions from a variety of DNA transfer systems. Nucleic Acids Res. 19, 3455. doi: 10.1093/nar/19.12.3455

Pansegrau, W., Lanka, E., Barth, P. T., Figurski, D. H., Guiney, D. G., Haas, D., et al. (1994). Complete nucleotide sequence of birmingham IncP $\alpha$ plasmids. J. Mol. Biol. 239, 623-663. doi: 10.1006/jmbi.1994.1404

Pansegrau, W., Schröder, W., and Lanka, E. (1993). Relaxase (TraI) of IncP alpha plasmid RP4 catalyzes a site-specific cleaving-joining reaction of singlestranded DNA. Proc. Natl. Acad. Sci. U.S.A. 90, 2925-2929. doi: 10.1073/pnas. 90.7.2925

Poulin-Laprade, D., Carraro, N., and Burrus, V. (2015). The extended regulatory networks of SXT/R391 integrative and conjugative elements and IncA/C conjugative plasmids. Front. Microbiol. 6:837. doi: 10.3389/fmicb.2015.00837

Sambrook, J., Fritsch, E. F., and Maniatis, T. (1989). Molecular Cloning: A Laboratory Manual. New York, NY: Cold Spring Harbor Laboratory Press.

Schultz, E., Barraud, O., Madec, J.-Y., Haenni, M., Cloeckaert, A., Ploy, M.C., et al. (2017). Multidrug resistance Salmonella genomic Island 1 in a Morganella morganii subsp. morganii human clinical isolate from France. mSphere 2:e00118-17. doi: 10.1128/mSphere.00118-17

Siebor, E., and Neuwirth, C. (2011). The new variant of Salmonella genomic island 1 (SGI1-V) from a Proteus mirabilis French clinical isolate harbours blaVEB-6 and qnrA1 in the multiple antibiotic resistance region. J. Antimicrob. Chemother. 66, 2513-2520. doi: 10.1093/jac/dkr335

Siebor, E., and Neuwirth, C. (2014). Proteus genomic island 1 (PGI1), a new resistance genomic island from two Proteus mirabilis French clinical isolates. J. Antimicrob. Chemother. 69, 3216-3220. doi: 10.1093/jac/dku314

Smith, C. J., and Parker, A. C. (1998). The transfer origin for Bacteroides mobilizable transposon Tn4555 is related to a plasmid family from grampositive bacteria. J. Bacteriol. 180, 435-439.

Solovyev, V., and Salamov, A. (2011). "Automatic annotation of microbial genomes and metagenomic sequences," in Metagenomics and Its Applications in Agriculture, Biomedicine and Environmental Studies, ed. R. W. Li (Hauppauge, NY: Nova Science Publishers), 61-78.

Swalla, B. M., Gumport, R. I., and Gardner, J. F. (2003). Conservation of structure and function among tyrosine recombinases: homology-based modeling of the lambda integrase core-binding domain. Nucleic Acids Res. 31, 805-818. doi: $10.1093 / \mathrm{nar} / \mathrm{gkg} 142$

Threlfall, E. J., Frost, J. A., Ward, L. R., and Rowe, B. (1994). Epidemic in cattle and humans of Salmonella Typhimurium DT 104 with chromosomally integrated multiple drug resistance. Vet. Rec. 134:577. doi: 10.1136/vr.134.22.577

Tsvetkova, K., Marvaud, J. C., and Lambert, T. (2010). Analysis of the mobilization functions of the vancomycin resistance transposon Tn1549, a member of a new family of conjugative elements. J. Bacteriol. 192, 702-713. doi: 10.1128/JB. 00680-09

Varsaki, A., Lucas, M., Afendra, A. S., Drainas, C., and De La Cruz, F. (2003). Genetic and biochemical characterization of $\mathrm{MbeA}$, the relaxase involved in 
plasmid ColE1 conjugative mobilization. Mol. Microbiol. 48, 481-493. doi: 10. 1046/j.1365-2958.2003.03441.x

Wisniewski, J. A., Traore, D. A., Bannam, T. L., Lyras, D., Whisstock, J. C., and Rood, J. I. (2016). TcpM: a novel relaxase that mediates transfer of large conjugative plasmids from Clostridium perfringens. Mol. Microbiol. 99, 884896. doi: $10.1111 / \mathrm{mmi} .13270$

Wozniak, R. A., Fouts, D. E., Spagnoletti, M., Colombo, M. M., Ceccarelli, D., Garriss, G., et al. (2009). Comparative ICE genomics: insights into the evolution of the SXT/R391 family of ICEs. PLoS Genet. 5:e1000786. doi: 10.1371/journal. pgen. 1000786

Zechner, E. L., Prüger, H., Grohmann, E., Espinosa, M., and Högenauer, G. (1997). Specific cleavage of chromosomal and plasmid DNA strands in gram-positive and gram-negative bacteria can be detected with nucleotide resolution. Proc. Natl. Acad. Sci. U.S.A. 94, 7435-7440. doi: 10.1073/pnas.94.14.7435

Ziegelin, G., Furste, J. P., and Lanka, E. (1989). TraJ protein of plasmid RP4 binds to a 19-base pair invert sequence repetition within the transfer origin. J. Biol. Chem. 264, 11989-11994.
Ziegelin, G., Pansegrau, W., Lurz, R., and Lanka, E. (1992). TraK protein of conjugative plasmid RP4 forms a specialized nucleoprotein complex with the transfer origin. J. Biol. Chem. 267, 17279-17286.

Zuker, M. (2003). Mfold web server for nucleic acid folding and hybridization prediction. Nucleic Acids Res. 31, 3406-3415. doi: 10.1093/nar/gkg595

Conflict of Interest Statement: The authors declare that the research was conducted in the absence of any commercial or financial relationships that could be construed as a potential conflict of interest.

Copyright (c) 2019 Kiss, Szabó, Hegyi, Douard, Praud, Nagy, Olasz, Cloeckaert and Doublet. This is an open-access article distributed under the terms of the Creative Commons Attribution License (CC BY). The use, distribution or reproduction in other forums is permitted, provided the original author(s) and the copyright owner(s) are credited and that the original publication in this journal is cited, in accordance with accepted academic practice. No use, distribution or reproduction is permitted which does not comply with these terms. 